\title{
A rice sitl1 mutant induced by gamma-ray irradiation shows enhanced insensitivity to salinity via reduced accumulation in $\mathrm{Na}+$ and $\mathrm{Mg} 2+$
}

\author{
Sung Don Lim ${ }^{1}$, Jong Ho Kim ${ }^{1}$, Jeongeun Lee ${ }^{1}$, Sung-Goo Hwang ${ }^{2}$, and Cheol Seong Jang ${ }^{1}$ \\ ${ }^{1}$ Kangwon National University \\ ${ }^{2}$ Sang Ji University
}

April 28, 2020

\begin{abstract}
Salinity stress is one of the most important abiotic stress factors affecting rice (Oryza sativa) production worldwide. Using a forward genetics approach, we isolated a salt insensitive TILLING line 1 (sitl1) rice mutant from a gamma-ray irradiated TILLING population. This sitl1 mutant line showed increased salinity tolerance due to reduced Mg2+ and Na+ uptake in root and shoot tissues and in the xylem sap. Whole genome resequencing and RNA-sequencing analyses identified a single nucleotide $\mathrm{T}$ insertion in exon 5 of Os01g47460 (putative metal ion transporter) with the CorA-like ZntB cation transfer domain (OsMGT1). Transient expression of the OsMGT1-sGFP fusion protein in rice protoplasts showed OsMGT1 was localized to the plasma membrane. Expression analysis of various tissues and at different growth stages showed that the OsMGT1 was mainly expressed in roots and leaf tissues as an early response to salinity stress. Complementation and overexpression assays in the yeast (Saccharomyces cerevisiae) CM66 strain and wild type showed that the OsMGT1 protein possessed both Mg2+ and Na+ transport activity. Taken together, the mutation of the OsMGT1 gene in the sitl1 mutant reduced the transport abilities of both $\mathrm{Na}+$ and $\mathrm{Mg} 2+$ conferring salinity insensitivity in rice.
\end{abstract}

\section{Introduction}

A doubling of global food production is likely necessary to meet the needs of a growing human population, which is expected to increase up to 9.3 billion by 2050 . However, abiotic stresses such as drought, salinity, and high temperatures are negatively impacting and exerting a drag on increasing the yield of major crops. Among them, salinity is the most important factor. Over 800 million hectares (6\%) of the land throughout the world and 45 million hectares $(20 \%)$ of irrigated land are affected by salinity resulting in a reduction of crop yield (). Most food crop species such as rice (Oryza sativa L.), wheat (Triticum aestivum L.), barley (Hordeum vulgare L.), and sorghum (Sorghum bicolor L.), etc. are glycophytes, therefore are not capable of growing in a saline environment. Of these crops, rice is highly sensitive to salinity, with a threshold of $3 \mathrm{dSm}^{-1}$ (deciSiemens per meter) electrical conductivity of saturated extract $\left(\mathrm{EC}_{\mathrm{e}}\right.$ ), which corresponds to approximately $30 \mathrm{mM} \mathrm{NaCl}$ for most cultivated varieties compared to $6-8 \mathrm{dSm}^{-1}(60-80 \mathrm{mM} \mathrm{NaCl})$ for wheat (Chinnusamy, Jagendorf, \& Zhu, 2005; Munns, 2005). This sensitivity is variable at different growth stages in rice (Munns \& Tester, 2008). In particular, rice is a more sensitive to salinity at the seedling stage, but it becomes moderately salinity insensitive at the tillering stage (Walia et al., 2005). Thus, even slightly higher salinity concentrations in soil than optimal levels can lead to retarded growth in rice plants at the seedling stage.

Roots are the first tissue exposed to salinity stress in soil and several genes have been identified along with their functional mechanisms to avoid the toxic effect of high salinity in roots. Both Salt Overlay Sensitive genes in rice (OsSOS1/OsNHX 7 ) andArabidopsis (AtSOS1/AtNHX 7 ) encode a plasma membrane 
$\mathrm{Na}^{+} / \mathrm{H}^{+}$antiporter, which have important roles in $\mathrm{Na}^{+}$extrusion in the roots under salinity conditions (Chinnusamy et al., 2005; Ding \& Zhu, 1997).AtSOS1 is mainly expressed in the root epidermis and xylem parenchyma and extrude the excess of $\mathrm{Na}^{+}$ions from root epidermal cells (Martinez-Atienza et al., 2007). Thus, theatsos1 mutants are salinity hypersensitive due to reduced rate of $\mathrm{Na}^{+}$extrusion resulting in increased $\mathrm{Na}^{+}$concentration in aerial parts of the atsos1mutant (Shi, Quintero, Pardo, \& Zhu, 2002). The salinity hypersensitive phenotype of the atsos 1 mutant was complemented by overexpression of the OsSOS1 in Arabidopsis indicating that both OsSOS1 and AtSOS1 participate in the root-shoot translocation of $\mathrm{Na}^{+}$ under salinity stress conditions (Shi, Ishitani, Kim, \& Zhu, 2000). After $\mathrm{Na}^{+}$uptake by the roots, a fraction of $\mathrm{Na}^{+}$is sequestrated into vacuoles through vacuolar $\mathrm{Na}^{+} / \mathrm{H}^{+}$antiporters of theAtNHX1 and OsNHX1, which are expressed in root, shoot, leaf, and flower tissues in Arabidopsis (Martinez-Atienza et al., 2007) and the stelar tissue, lateral roots, and vascular bundles in the shoot of rice seedlings, respectively (Apse, Aharon, Snedden, \& Blumwald, 1999; Fukuda, Nakamura, Hara, Toki, \& Tanaka, 2011). Thus, these two $\mathrm{Na}^{+} / \mathrm{H}^{+}$antiporters of SOS1 and NHX1 are involved in critical roles of $\mathrm{Na}^{+}$exclusion and sequestration to reduce salinity toxicity in plant, respectively.

Several ion channels and carrier-type transporters also have been identified whose functional roles involve $\mathrm{Na}^{+}$uptake in plants. Among these, the HKT family is quite diverse in functions and well-characterized in several crop species. This HKT family is further divided into two distinct classes (HKT1 and HKT2) based on their transport characteristics (Almeida, Oliveira, \& Saibo, 2017; Fukuda et al., 2004; Platten et al., 2006). Most members of class I transporters, HKT1s, including AtHTK1;1 in Arabidopsis (Maser et al., 2002; Sunarpi et al., 2005), OsHKT1;1 , OsHKT1;3 ,OsHKT1;4 and OsHKT1;5 in rice (Berthomieu et al., 2003; Cotsaftis, Plett, Shirley, Tester, \& Hrmova, 2012; Jabnoune et al., 2009), and TaHKT1;4 and TaHKT1;5 in wheat (Byrt et al., 2007; Ren et al., 2005) have been implicated in controlling $\mathrm{Na}^{+}$accumulation in shoot as $\mathrm{Na}^{+}$selective exclusion transporters for enhancing salinity stress tolerance. The class II transporters of HKT are only found in monocot species (Huang et al., 2006). All members of identified HKT2 including OsHKT2;1 and OsHKT2;2 in rice (Platten et al., 2006; Yao et al., 2010), TaHKT2;1 in wheat (Horie et al., 2007), and HvHKT2;1 in barley (Schachtman \& Schroeder, 1994) are clearly shown to be involved in mediating $\mathrm{Na}^{+}$influx in root tissues under $\mathrm{K}^{+}$starvation conditions. Although the mechanisms regulating the transport activity of the most HKT genes are unknown, one magnesium transporter has been reported, OsMGT1 (OsMRS2-1) protein is involved in enhancing OsHKT1;5 activity in rice (Mian et al., 2011).

Magnesium ion $\left(\mathrm{Mg}^{2+}\right)$ is one of the most abundant free divalent cations and essential macronutrient for plants. $\mathrm{Mg}^{2+}$ is essential for photosynthesis as a central metal for chlorophylls and acts as a cofactor for structural conformation for many enzymes in catalytic processes (Chen et al., 2017). Thus, $\mathrm{Mg}^{2+}$ deficiency in plants generally results in a reduction of root and shoot growth and necrosis in leaves due to the decline of chlorophyll and carbon fixation (Hermans et al., 2010; Shaul, 2002). Several ionomic analysis showed that $\mathrm{Mg}^{2+}$ concentrations decreased significantly with increasing $\mathrm{Na}^{+}$levels in many plant species including rice (Hakim et al., 2014; Hermans \& Verbruggen, 2005; Munns \& Tester, 2008; Talei, Kadir, Yusop, Valdiani, \& Abdullah, 2012; Yildirim, Karlidag, \& Turan, 2009). This reduced $\mathrm{Mg}^{2+}$ uptake might be due to the suppressive effect of $\mathrm{Na}^{+}$or transport activities of $\mathrm{Na}^{+}$and $\mathrm{Mg}^{2+}$ transport could compete with each other under salinity stress condition, but actual mechanisms remain unclear. Alternately, there is $\mathrm{Na}^{+} / \mathrm{Mg}^{2+}$ antiporter which plays a major role in $\mathrm{Mg}^{2+}$ extrusion in humans (Akter \& Oue, 2018), however, this antiporters have not yet been discovered in plants. The bacterial CorA protein and its yeast homologs of CorA-type transporters of Alr1 and Mrs2 proteins are well characterized as $\mathrm{Mg}^{2+}$ transporters in all living organisms (Sontia \& Touyz, 2007). In plants, there are 11 AtMRS2/MGT and 9 OsMRS2/MGT homologs of bacterial CorA-type transporter in Arabidopsis and rice, respectively (Knoop, Groth-Malonek, Gebert, Eifler, \& Weyand, 2005; L. Li, Tutone, Drummond, Gardner, \& Luan, 2001; Saito et al., 2013). The CorAtype MRS2/MGT proteins have a unique topology with two C-terminal transmembrane (TM) domains and the conserved Gly-Met-Asn (GMN) tripeptide motif is located at the end of the first TM domain that is thought to be essential for $\mathrm{Mg}^{2+}$ transport activity. A distant CorA homolog in Salmonella typhimurium (ZntB), which alters the GMN-motif to GIN-motif, has been reported as putative zinc transporter involved in $\mathrm{Zn}^{2+}$ and $\mathrm{Cd}^{2+}$ transport activity (Knoop et al., 2005; Schock et al., 2000). However, homologs of CorA-like 
ZntB transporters in plant species have not reported for their exact roles.

\section{Materials and methods}

\section{sitl1 mutant screening and growth condition}

To screen for salinity tolerance mutant lines, seeds of the $\mathrm{M}_{10}$ core collection germplasms derived from the application of gamma-ray irradiation and wild-type (WT) rice (Oryza sativa, cv. Donganbyeo) were germinated on moistened filter paper at $28{ }^{\circ} \mathrm{C}$ for $2 \mathrm{~d}$. The germinated seeds were transferred to a nylon net stretched over a floating Styrofoam in a plastic container containing half-strength Kimura B nutrient solution (NS) at pH 5.6 (Worlock \& Smith, 2002), which was renewed every two days in a growth chamber under a $16 \mathrm{~h} / 8 \mathrm{~h}$ (light/dark) cycle at $28 / 23{ }^{\circ} \mathrm{C}$. At the three-leaf stage (7-day-old), rice seedlings were treated with nutrient solution containing $100 \mathrm{mM} \mathrm{NaCl}$ for one more week. The lengths of shoots and roots for each individual plant were measured with 3 biological replicates.

\section{Phenotype analyses of sitl1 mutant}

For the seed germination assays, seeds of the sitl1 and WT were placed on moistened filter paper in a growth chamber under 16 -h photoperiod. Seeds germination rates were scored every day for a week after sowing. To analyze phenotypic difference of the sitl1 and WT, plants were grown in the half-strength NS or double distilled water (DW). Plants were used to quantify the length and fresh weight of the coleoptile, root, leaf sheath, and leaf blade. To observe detailed root morphology, detached roots of the sitll and WT grown in NS or DW were scanned at $7 \mathrm{~d}$ after germination, and primary root lengths and widths, lateral root lengths, widths, numbers, and density in mature root zone $(3 \sim 4 \mathrm{~cm}$ away from root tip) were quantified using ImageJ software (http://imagej.nih.gov/ij/).

\section{Leaf chlorophyll assay}

Homogenized leaf samples (300 mg) of the sitl1 and WT were used to determine chlorophyll content. Samples were incubated in $5 \mathrm{~mL}$ of $80 \%$ acetone in the dark for $30 \mathrm{~min}$. After centrifugation at $15000 \times \mathrm{g}$ for $15 \mathrm{~min}$, supernatants were transferred to a 96-well microplate. The absorbance was measured at 645 and $663 \mathrm{~nm}$ using a SPECTROstarNano (BMG labtech, Germany) and chlorophyll a, b, and total chlorophyll content were calculated as described (Ma, Goto, Tamai, \& Ichii, 2001).

\section{Root cell size and number analysis}

Seeds of the sitl1 and WT were germinated and grown in half-strength NS in a growth chamber under a 16-h photoperiod for 1 week. The detached roots were incubated in Propidium iodide (PI, Sigma-Aldrich, USA) at a final concentration of $10 \mu \mathrm{M}$ for $10 \mathrm{~min}$. Samples were rinsed three times with sterile deionized water to remove unbounded PI, and mounted in $25 \%$ (v/v) glycerol on glass microscope slides. Images of root meristem and root cells in the mature zone were captured at $561 \mathrm{~nm}$ and $642 \mathrm{~nm}$ for excitation and emission, respectively, via a confocal laser-scanning microscope (LSM 880, Carl Zeiss, Germany). Cell length, width, and area of the root meristem zone and in the mature root zone were determined using ImageJ software as described (Arnon, 1949; Lim et al., 2018).

\section{Salinity and drought stress treatments}

For the salinity stress treatment, 1-week-old seedlings were treated with different concentrations of $\mathrm{NaCl}$ solution $(0,50$, or $100 \mathrm{mM})$ for 1 week. Fresh weights of root, leaf sheath, and leaf blade tissues were measured at 1 week after $\mathrm{NaCl}$ treatments. For the salinity stress treatment under soil-grown condition, 1-week-old seedlings were irrigated with $50 \mathrm{mM} \mathrm{NaCl}$ solution for 2 weeks. For the drought stress, 1-week-old seedlings were withheld irrigation for 1 week and rewatered for 1 week. Fresh weights of aerial shoots after salinity and drought stress treatment were measured.

\section{Hydrogen peroxide $\left(\mathrm{H}_{2} \mathrm{O}_{2}\right)$ analysis}

To observe $\mathrm{H}_{2} \mathrm{O}_{2}$ production in leaf tissues of the sitl1 and WT, histochemical DAB staining was conducted as described (Hacham et al., 2011). After salinity stress treatment for 1 weeks, detached leaves were incubated 
in DAB (3,3'-diaminobenzidine) staining solution at $25{ }^{\circ} \mathrm{C}$ for $5 \mathrm{~h}$. Bleaching solution was used to remove chlorophylls of the leaf samples and images were captured by light microscopy (DM500, Leica, Germany). $\mathrm{H}_{2} \mathrm{O}_{2}$ content in root and leaf tissues was quantitated as described in Gay and Gebicki (2012). The absorbance was measured at $560 \mathrm{~nm}$ using a SPECTROstarNano. A standard curve of $\mathrm{H}_{2} \mathrm{O}_{2}$ using linear regression analysis was used to calculate the sample $\mathrm{H}_{2} \mathrm{O}_{2}$ concentration.

\section{$\mathrm{Na}^{+}$distribution analysis}

To visualize the $\mathrm{Na}^{+}$distributions in the lateral roots and leaf protoplasts of sitl1 and WT, a $\mathrm{Na}^{+}$-specific fluorescent dye, CoroNa-Green AM (Invitrogen, USA) was used (Gay \& Gebicki, 2000). For the lateral root staining, seedlings were grown under half-strength NS for 1 week and treated with $50 \mathrm{mM} \mathrm{NaCl}$ for $3 \mathrm{~h}$. Each root was detached and washed 3 times with distilled water and stained with $50 \mu \mathrm{M}$ CoroNa-Green AM for $16 \mathrm{~h}$. Samples were washed again and incubated with the lipophilic probe FM4-64 (Thermo Fisher Scientific, USA) for $5 \mathrm{~h}$ and re-washed 3 times. Images of the intercellular $\mathrm{Na}^{+}$fluorescence and vacuolar membrane were captured using a confocal microscope.

For the $\mathrm{Na}^{+}$distribution in protoplasts, the leaf protoplasts were isolated from 1-week-old rice seedlings as described (Park, Lee, Lee, Byun, \& Kim, 2009). After the enzymatic digestion, WI buffer (0.5 M mannitol, 4 $\mathrm{mM}$ MES, and $20 \mathrm{mM} \mathrm{KCl}, \mathrm{pH} 5.7)$ was used to avoid the effect of a high concentration of $\mathrm{Na}^{+}$in W5 buffer for protoplast incubation (Lim, Lee, \& Jang, 2014). Isolated protoplasts were incubated with WI buffer containing 0 and $50 \mathrm{mM} \mathrm{NaCl}$ for $1 \mathrm{~h}$ and then washed 3 times with WI buffer. The CoroNa-Green AM was added to the samples for a final concentration of $10 \mu \mathrm{M}$. After $5 \mathrm{~h}$, the protoplasts were re-washed with WI buffer and the fluorescence was captured using a confocal microscope. CoroNa-Green AM and FM4-64 were excited at $488 \mathrm{~nm}$ and $543 \mathrm{~nm}$ with a laser, respectively, and the fluorescence emission was collected at 510 $\mathrm{nm}$ (range 50nm) for CoroNa-Green and $612 \mathrm{~nm}$ (rage $50 \mathrm{~nm}$ ) for FM4-64. Average fluorescence intensity of the CoroNa-Green in the cytosol and the vacuole was measured using imageJ software as described (Zhang et al., 2012).

\section{Ionomic analysis}

Hydroponically grown 1-week-old plants were treated with NS containing 0 or $50 \mathrm{mM} \mathrm{NaCl}$ for 1 week in a growth chamber under a 16-h photoperiod. Samples were harvested and oven-dried at $80{ }^{\circ} \mathrm{C}$ for $16 \mathrm{~h}$. Dried tissue samples were used to determine ion elements of $\mathrm{K}, \mathrm{Mg}, \mathrm{Na}, \mathrm{Ca}$, and $\mathrm{P}$ by inductively coupled plasmaoptical emission spectroscopy (ICP-OES, Optima 700 dv, Perkin Elmer, USA) at the Central Laboratory, Kangwon National University. Xylem sap was collected from 1-week-old rice seedlings grown in half-strength NS. One hour after starting the 0 or $50 \mathrm{mM} \mathrm{NaCl}$ treatments, xylem sap exuded from the cut surface of leaf sheath was collected by micropipette into a $1.5 \mathrm{ml}$ E-tube. The amount of collected sap was weighted and the $\mathrm{Mg}, \mathrm{Na}$, and $\mathrm{K}$ ions were determined.

\section{Whole genome sequencing (WGS) and RNA-sequencing analyses}

The genomic DNA was isolated from leaf samples of each plant using the CTAB method (Saghai-Maroof et al., 1984). The Illumina TruSeq(r) Nano DNA Library Kit (Illumina, USA) was used to construct the DNA libraries. WGS was performed using the Illumina HiSeq4000 platform (Macrogen Inc., Korea) with a paired-end sequencing method. The sequencing depth of WGS was 137x coverage for WT and 24x coverage for the sitl1 . The short reads were filtered by NGS QC Toolkit (Lim, Kim, Gilroy, Cushman, \& Choi, 2019). The high-quality reads were $85.45 \%$ for WT and $84.03 \%$ for sitl1 after filtering (Figure S1a). The PCR duplicates were removed via Samtools (http://www.htslib.org/). A total number of 298,835,366 reads for WT and 55,182,756 reads for the sitl1 were obtained (Figure S1b). Approximately 96\% of obtained reads were mapped on the Nipponbare reference genome (Os-Nipponbare-Reference-IRGSP-1.0) from the RAP-DB database (https://rapdb.dna.affrc.go.jp/) by Burrows-Wheeler aligner (Patel \& Jain, 2012). The properly paired reads were $95.78 \%$ for WT and $95.92 \%$ for the sitl1 . The variant calling was performed in the GATK program with HaplotypeCaller (https://gatk.broadinstitute.org/hc/en-us). We removed the lower quality variants (missing variants, GQ value $<21$, Phred Quality score $<21$, missing rate $>0.4$, and non-pass variants detected by VQSR). 
The RNA-seq was performed using Illumina HiSeq4000 platform with 3 independent biological replicates. The high-quality reads were obtained from the raw data of RNA-seq as described above. Approximately $99 \%$ of high-quality short reads were obtained (Figure S1c) and the reads were assembled by using Cufflinks (http://cole-trapnell-lab.github.io/cufflinks/). The Cuffdiff was used to determine DEGs, which had the statistical significance with q value less than 0.05 . To survey the overrepresented metabolism and gene expression patterns, we used the MapMan program (https://mapman.gabipd.org/).

\section{Molecular cloning and subcellular localization analyses}

To identify the T insertion mutation in OsMTP1 gene, genomic DNA was extracted using DNeasy Plant Mini Kit (Qiagen, USA) and target region was amplified using Q5 DNA polymerase (NEB, USA) with appropriate primers (Table S1). For cloning the full-length coding sequence of theOsMTP1 gene, total RNA was extracted from 1-week-old seedlings of the sitl1 and WT using RNeasy Plant Mini Kit (Qiagen). Firststrand cDNA was synthesized using the $1^{\text {st }}$ strand cDNA synthesis kit (Takara-Bio, Otsu, Japan) with oligo dT and Q5 DNA polymerase was used to amplify the full-length OsMTP1 gene. The PCR-amplified product was purified and cloned into the directional $\mathrm{TOPO}^{(\mathrm{r})}$ vector (Invitrogen). The plasmids were extracted and verified by Sanger DNA sequencing.

For the subcellular localization, the OsMTP1 gene was cloned into the binary vector ImpGWB405 (H. Li \& Durbin, 2009) containing the C-terminal sGFP using the Gateway LR Clonase II Enzyme Mix (Invitrogen). For transient expression, $10 \mu \mathrm{g}$ of each35S::OsMTP1-sGFP , 35S::sGFP (Nakagawa et al., 2007), and pm-rk (CD3-1007) (Lim et al., 2018) was transfected into rice protoplasts using a 40\% PEG solution as described (Nelson, Cai, \& Nebenfuhr, 2007). Transfected protoplasts were incubated overnight in the dark and then subcellular localization was observed using a confocal microscope.

\section{Gene expression analysis}

Various tissue samples of germinated seed, roots, leaf sheath, leaf blade, node, panicle, and immature seeds were harvested from different development stages (1-, 3-, and 16-week-old) of WT plants. To evaluate salinity stress induced changes in gene expression, 1-week-old seedlings were treated with 0 and $50 \mathrm{mM} \mathrm{NaCl}$ solution and root and tissue samples were harvested at $30 \mathrm{~min}, 1 \mathrm{~h}, 6 \mathrm{~h}$, and $24 \mathrm{~h}$ after treatment. Total RNA was extracted using TRIzol@ reagent (Ambion, USA) and treated with DNAse I (Sigma-Aldrich) according to manufacturer's instructions. One microgram of the total RNA was used to synthesize first strand cDNA using random oligomers. Real-time qPCR was performed using SYBR® Green TOP real qRT-PCR PreMIX (EnzynomicsTM, Daejeon, Korea), and the SYBR signals were monitored using a C1000 Thermal Cycler and CFX96 Real-Time System detection instrument (Bio-Rad Laboratories, USA). Expression levels of the OsMTP1 and selected marker genes (Lim et al., 2014) calculated using the $2^{-\Delta \Delta^{\circ} \mathrm{T}}$ method (Islam et al., 2017). OsActinII was used as an internal control (Livak \& Schmittgen, 2001).

\section{Yeast complementation assay}

$\mathrm{Mg}^{2+}$ transport ability of OsMTP1 protein was tested by complementation of a yeast CM66 mutant, which lacks both $\mathrm{Mg}^{2+}$ transporters of ALR1 and ALR2 gene, and CM52 (Bi et al., 2009). Full-length coding sequence of the OsMTP1 andmOsMTP1 (mutated OsMTP1) were PCR-amplified and ligated into BamHI/SalI-digested pRS425-ADH1 p plasmid. The plasmid was transformed into Escherichia coli and the ADH1p::OsMTP1 and ADH1p::mOsMTP1 plasmids were transformed into CM66 and CM52 yeast cell lines on standard synthetic media lacking leucine (SD-LEU) supplemented with $10 \mathrm{mM} \mathrm{MgCl} 2$. The transformed yeast cell lines were cultured in liquid SD-LEU and adjusted to $1.0 \mathrm{OD}_{600}$, diluted in a 10 -fold series, with sterilized water. Ten microliter of the yeast cells were spotted on solid SD-LEU media containing 0, 0.1, or 1 of $\mathrm{MgCl}_{2}$. For the liquid media assay, growth rates of each transformant was monitored for $66 \mathrm{~h}$ using a SPECTROstarNano.

The $\mathrm{Na}^{+}$transport ability of OsMTP1 protein was examined using wild-type yeast strain FM391 (MA-

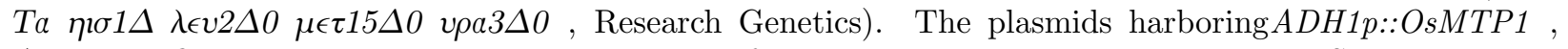
ADH1p::mOsMTP1, and empty vector were transformed into the FM391 and selected on SD-LEU media. 
The transformants were cultured in complete media (YPD) and spotted on solid YPD media containing 0, 0.5 , or $1 \mathrm{M}$ of $\mathrm{NaCl}$ and $1 \mathrm{M}$ of $\mathrm{NaCl}$ with 0.1 or $1 \mathrm{mM} \mathrm{MgCl}_{2}$. The liquid media assay was conducted for $48 \mathrm{~h}$ under same conditions described above.

\section{Results}

\section{sitl1 mutant decreases root growth and leaf chlorophyll content}

Previously, we have developed 15,000 fertile $\mathrm{M}_{3}$ TILLING rice mutant lines (Oryza sativa, cv Donganbyeo) via the application of gamma-ray irradiation with 200 and 300 Gy (Hwang, Hwang, Kim, \& Jang, 2014; L. Li et al., 2001). From this TILLING population, we selected 100 mutant lines based on evaluation on abiotic stresses, and subsequently developed $\mathrm{M}_{10}$ homozygous lines. This core collection was used to screen germplasm collections for seedling-stage salinity tolerance. Of these, one mutant line (300Gy-1011) showed significantly increase in both shoot and root growth compared to wild-type (WT) plants after salt treatment (Figure S2). This mutant line was named salt-insensitive TILLING line 1(sitl1) and was selected for further phenotypic analysis.

In order to evaluate phenotype differences between sitl1 and WT, plants were grown in hydroponic solution and root and shoot growth was monitored for 3 weeks. Although plants showed no significant difference in germination rate (Figure S3), the sitl1 showed significantly decreased root growth as measured by either length or fresh weight of root tissues in seedlings (Figure 1a). The sitl1 showed a 0.79-fold decrease in root length, and 0.84-fold decrease in root fresh weight, relative to WT (Figure 1b, c). However, no significant differences were observed in lengths and fresh weights of coleoptile, leaf sheath, or leaf blade between the sitl1 and WT plants. We also noticed that leaves of the sitl1 were slightly paler green compared to WT in seedlings (Figure 1d). In addition, relatively old leaves $\left(2^{\text {nd }}\right.$ and $3^{\text {rd }}$ leaf) of thesitl1 displayed an accelerated leaf senescence phenotype compared to WT at the same development stage (Figure 1d). Consistent with the observed leaf color, leaf chlorophyll content of the sitl1 were significantly less than that of WT (Figure 1e). The observed decreases in root growth and leaf chlorophyll content of the sitl1 did not appear when the sitl1 was grown in DW (Figure 1f-i).

Next, we tested whether the observed phenotypes in the sitl1 is potentially linked to $\mathrm{Mg}^{2+}$ deficient symptoms. Seedlings were grown in nutrient solution containing various levels of $\mathrm{Mg}^{2+}$ ranged from 0 to $500 \mu \mathrm{M}$. Although, thesitl1 exhibited root growth defects in the solution containing $0,10,100 \mu \mathrm{M}$ of $\mathrm{Mg}^{2+}$, the root growth inhibition was rescued in the solution containing $500 \mu \mathrm{M}$ of $\mathrm{Mg}^{2+}$ (Figure S4a,b). Fresh weights of aerial shoot between the sitl1 and WT were not significantly different under low $\mathrm{Mg}^{2+}$ conditions, but the sitl1 had significantly higher shoot weight in the solution containing $500 \mu \mathrm{M}$ of $\mathrm{Mg}^{2+}$ (Figure S4c). We also observed reduced total chlorophyll content in the leaves of sitl1 which was rescued when the solution $\mathrm{Mg}^{2+}$ levels reached to $500 \mu \mathrm{M}$ (Figure S4d,e). These results suggest that the sitl1 might have less ability to uptake $\mathrm{Mg}^{2+}$ ions, which affects root growth, leaf senescence, and chlorophyll synthesis.

\section{sitl1 mutant alters root development}

The root morphology analysis revealed a significant decrease in lateral root number, average lateral root length, and sum of lateral root length but had no significant change on lateral root density in thesitl1 compared to WT under normal condition (Figure 2a-e). In addition to the primary root growth, the lengths of root immature zone and mature zone in the sitl1 decreased by 0.79 -fold and 0.77 -fold compared to WT, respectively. Also, the primary root width of thesitl1 decreased but the lateral root width was not significantly changed compared to WT (Figure 2h,i). Under the deionized water-grown condition, all significant changes in the root growth of sitl1 were not observed (Figure 2b-i).

To determine whether the reduction in primary root growth was due to decreased cell proliferation or cell elongation in the sitl1, the root cell number and the length of epidermis in the apical meristem and the basal meristem were compared between the sitl1 and WT (Figure 3). The measurement of the cell number in root meristem zone showed that the average cell number in the apical meristem significantly decreased by 0.87-fold in the sitl1 but cell number in the basal meristem was not changed compared to that of WT (Figure 
3b,c). Total cell number in root meristem was 0.9-fold lower than WT (Figure 3d). The length of the apical, basal and total meristem in the sitl1 was 0.91-, 0.78-, and 0.82-fold shorter than that in the WT, respectively (Figure 3e-g). These decreases in length were due in part to a decrease in cell number within the apical meristem (Figure 3b,e). Next, cell length was measured to verify if the decreased basal meristem length in thesitl1 was due to slow rates of the cell elongation within the basal meristem zone. Indeed, average cell length and width in the basal meristem, and average cell width in the apical meristem decreased by 0.75-, $0.79-$, and 0.75 -fold respectively, whereas average cell length in the apical meristem was not significantly changed in the sitl1 compared to WT (Figure 3h,i). Furthermore, individual cell size (e.g. length, width and area) of the root exodermal cell in the mature root zone also decreased significantly, relative to control lines (Figure 3j-m). These results suggest that the reduced ability of $\mathrm{Mg}^{2+}$ uptakes in the sitl1 likely contributes to the decreased the rate of cell proliferation and cell elongation resulting in growth reduction in root tissues.

\section{sitl1 mutant enhances salinity stress insensitivity}

sitl1 mutant showed significantly decreased root biomass and leaf chlorophyll content when plants were grown in NS (Figure 4a-e). In contrast, when plants were treated with NS containing 50 and $100 \mathrm{mM} \mathrm{NaCl}$, the sitl1 produced 1.22-fold, 1.19-fold, and 1.26-fold more biomass in root, leaf sheath, and leaf blade than WT, respectively (Figure 4b). Theses increased root and shoot biomass in the sitl1 was also observed when plants were treated with DW containing 50 and $100 \mathrm{mM} \mathrm{NaCl}$ (Figure 4c). The sitl1 also had higher chlorophyll content than the WT plant when plants were subjected to the treatment with NS or DW containing 50 and $100 \mathrm{mM} \mathrm{NaCl}$ (Figure 4d,e). We further confirmed that the sitl1 showed improved salinity insensitivity when plants were irrigated with $50 \mathrm{mM} \mathrm{NaCl}$ solution under soil-grown conditions but the sitl1 did not show drought stress insensitivity (Figure S5). These results confirm that the sitl1 have a higher ability to tolerate salinity stress but not drought stress.

In order to visualize and quantitate the $\mathrm{H}_{2} \mathrm{O}_{2}$ accumulations, we conducted histochemical DAB staining and $\mathrm{H}_{2} \mathrm{O}_{2}$ quantification analysis at 7 days after treatment with $0,50,100 \mathrm{mM} \mathrm{NaCl}$ (Figure 4f-h). Under unstressed condition, no significant differences were observed in the amounts of $\mathrm{H}_{2} \mathrm{O}_{2}$ between the root tissues of thesitl1 and WT, whereas the sitl1 line showed a 1.47-fold increase in $\mathrm{H}_{2} \mathrm{O}_{2}$ amount in leaf tissues compared to the WT (Figure $4 \mathrm{~g}-\mathrm{h})$. Under salinity stress condition, thesitl1 showed a $0.45-(50 \mathrm{mM} \mathrm{NaCl})$ to 0.5 -fold $(100 \mathrm{mM} \mathrm{NaCl})$ decrease in root tissues, and $0.53-(50 \mathrm{mM} \mathrm{NaCl})$ to 0.58 -fold $(100 \mathrm{mM} \mathrm{NaCl})$ decrease in leaf tissues, respectively, relative to the WT (Figure 4f-h).

Sodium ion concentration within the lateral roots was measured using the cell-permeant CoroNa Green AM dye, which is a green-fluorescent $\mathrm{Na}^{+}$-specific indicator that exhibits an increase in fluorescence emission intensity upon binding $\mathrm{Na}^{+}$levels (Cho, Park, Kim, \& Jang, 2010). Seven-day-old seedlings were transferred to nutrient solution containing $50 \mathrm{mM} \mathrm{NaCl}$ for $3 \mathrm{~h}$. The salinity-treated WT plants showed strong fluorescence signals both in the vacuoles and the cytoplasm of outer epidermis and inner cells, whereas the sitl1 showed very little fluorescence signals, which tightly sequestrated into the vacuoles in roots (Figure 4i).

In order to validate the ability of $\mathrm{Na}^{+}$influx across the plant plasma membranes, we used freshly isolated protoplasts from leaf tissues because it was observed the difference in root development of the sitl1 could alter the apoplastic permeability of $\mathrm{Na}^{+}$or the ability of root apopolastic barriers, which block $\mathrm{Na}^{+}$transport to shoot tissues in rice (Meier, Kovalchuk, \& Rose, 2006; Zhou et al., 2011). The salinity-treated WT protoplasts showed obvious CoroNa Green AM staining, whereas the protoplasts of the sitl1 showed very little staining (Figure 4j). Quantitation of the CoroNa-Green AM intensity showed that the WT displayed significantly higher fluorescence than the sitl1 in the vacuole and the cytoplasm. Under unstressed conditions, there was no significant differences in fluorescence intensity. However, under $50 \mathrm{mM} \mathrm{NaCl}$, the WT protoplast showed 6.95-fold and 4.15-fold higher fluorescence intensity than the sitl1 , in the cytoplasm and in the vacuoles, respectively (Figure 4k). These results demonstrate that the improved salinity tolerance of the sitl1 caused major changes such as reduced $\mathrm{Na}^{+}$influx rate across the plasma membrane in both root and leaf tissues.

\section{sitl1 mutant reduces $\mathrm{Na}^{+}$and $\mathrm{Mg}^{2+}$ uptake and root-to-shoot translocation}

In order to investigate the mechanistic basis of salinity tolerance and the observed reduction in root devel- 
opment and leaf chlorophyll content, the ion concentrations of the sitl1 and WT plants grown for 1 week under $0 \mathrm{mM}$ or $50 \mathrm{mM} \mathrm{NaCl}$ stress conditions were measured (Figure 5a-e). Under unstressed conditions, $\mathrm{K}$ content was increased by 1.38 -fold and $\mathrm{Mg}$ was reduced by 0.61 -fold, respectively, in the root of sitl1 compared to that of WT (Figure 5a,b). Mg content in leaf tissues was also reduced by 0.76-fold in sitl1 , whereas, $\mathrm{Na}, \mathrm{Ca}$, and $\mathrm{P}$ content were not changed in both root and leaf tissues (Figure 5c-e). Under salinity stress conditions, $\mathrm{K}$ content was increased 1.75-fold, however, $\mathrm{Mg}$ and $\mathrm{Na}$ content were reduced 0.82 -fold and 0.67 -fold, respectively, in the root of sitl1 . Notably, significant reduction of $\mathrm{Mg}$ and Na content were observed in both roots and leaves of the sitl1 under $100 \mathrm{mM} \mathrm{NaCl}$ treatment (Figure 5b,c).

Next, we analyzed $\mathrm{Mg}, \mathrm{Na}$, and K concentrations in xylem sap (Figure 5a-e). The Mg and Na concentrations in xylem sap were significantly reduced 0.56-fold and 0.64-fold, respectively, compared to the WT, whereas K concentration in xylem sap was not changed under normal condition (Figure $5 \mathrm{f}-\mathrm{h}$ ). Mg and Na concentrations in xylem sap were also significantly lower in the sitl1 than in the WT under $100 \mathrm{mM} \mathrm{NaCl}$ stress (Figure $5 f, g)$. Collectively, these results demonstrate that improved salinity tolerance, and reduced root growth and leaf chlorophyll content in sitl1 caused major changes to the $\mathrm{Mg}$ and $\mathrm{Na}$ content in both roots and leaves.

\section{Gene expression profiling of antioxidant defense enzymes and $\mathrm{Na}^{+}$and $\mathrm{K}^{+}$transporters}

To better understand the salinity insensitive mechanism in thesitl1, the transcription abundance of genes encoding antioxidant enzymes were analyzed using quantitative qRT-PCR (Figure S6 and Figure 6). The expression levels of antioxidant enzyme genes were not changed significantly between the sitl1 and WT under normal condition. However, the transcript abundance of the OsCAT1 showed 0.14- and 0.4-fold decrease in the root and leaf tissues of sitl1 (Figure 6a), respectively, compared to WT plants. Under salinity stress conditions, most antioxidant enzyme genes were strongly upregulated in both root and leaf tissues of the WT plants, whereas in thesitl1, the genes did not show the upregulated expression levels, especially in leaf tissues. For examples, gene expression levels of OsCAT2 , OsAPX1 , OsAPX2, OsPOD , OsGR1 ,OsGR2 , OsDHAR1, and OsMDHAR2 were highly increased in leaf tissues of WT upon salinity stress. However, the expression levels of these genes in sitl1 showed only 1.2-, 1.2-, 1.2-, 1.3-, 1.7-, 2.3-, 1.6-, or 3.0- fold inductions, respectively (Figure 6a). Furthermore, we confirmed that 8 out of 15 antioxidant enzyme genes showed significant decreases in transcript abundance in both root and leaf tissues of the sitl1 compared to WT.

Alterations in $\mathrm{Na}^{+}$and $\mathrm{K}^{+}$transporter functions were explored by comparing their transcript abundance in the sitl1 and WT. OsLti6a and OsLti6bgenes encode low-molecular weight membrane proteins required for the prevention of excess $\mathrm{Na}^{+}$entry in the cell (Krishnamurthy, Ranathunge, Nayak, Schreiber, \& Mathew, 2011). Transcript levels of OsLti6a and OsLti6b did not show any difference between the sitl1 and WT (Figure 6b). The expression of OsHKT1;5 was higher (2-fold) in the root tissues of thesitl1 compared to WT under normal condition (Figure S6c and Figure 6b). This increased transcript levels of OsHKT1;5 in thesitl1 were maintained when plants were subjected to salinity stress. In contrast, the expression of OsHKT2;1 did not change in both plants under normal condition. Under salinity stress condition, theOsHKT2;1 showed opposite expression patterns in both tissues. TheOsHKT2;1 in sitl1 showed decreased (0.5-fold) transcript accumulation in roots and increased $\left(3.5\right.$-fold) transcription accumulation in leaves. A vacuolar $\mathrm{Na}^{+} / \mathrm{H}^{+}$ antiporter, OsNHX1, showed decreased transcript abundance in both roots and leaves in thesitl1. The transcript abundance of OsSOS1 increased in the roots of sitl1 under normal conditions but decreased transcription levels were observed in both tissues under salinity stress condition. Another known $\mathrm{Na}^{+}$influx channel of OsCNGC1 also showed significantly reduced transcript abundance in both tissues of the sitl1 under salinity stress condition. Lastly, OsAKT1 and OsHAKr genes showed decreased transcript abundance in both tissues of the sitl1 under salinity stress condition.

\section{Identification of OsMTP1 as a possible element responsible for the sitl1 mutant}

To identify the causal mutations in the sitl1 line, whole genome sequencing (WGS) was performed. A total of 116,584 SNPs and 13,196 Indels were identified as shared variants between WT (Oryza sativa, cv. Donganbyeo) and sitl1 based on rice reference genome (Oryza sativa, cv. Nipponbare) (Figure S7). In 
addition, a total of 435 SNPs and 195 Indels were not shared between sitl1 and WT genomes, indicating that these nonshared variants were caused by gamma-ray irradiation in the sitl1 (Figure 7a). To survey the effects of variants on the sitl1 genome, the variants were divided into the two groups, the genic region and the nongenic region according to their genome locations. Most variants of SNPs (87\%) and Indels (78\%) in the sitl1 were located in the nongenic region (Figure 7b). Fifty-seven out of 435 nonshared SNPs (13\%) and 43 out of 195 Indels $(22 \%$ ) were located in the genic region (Figure $7 \mathrm{~b}$, left panel). Among them only 21 SNPs and 13 Indels were detected in the coding sequence (CDS) region of the genes (Figure 7b). The 21 SNPs located in the CDS regions included 12 missense, 8 synonymous, and 1 stop-gained variants (Figure $7 \mathrm{~b})$. The 13 Indels located in the CDS regions included 1 inframe insertion, 2 inframe deletion, 1 splice acceptor, 7 frameshift, and 2 stop-gained variants (Figure $7 \mathrm{~b}$ ).

To investigate the transcriptional changes driving the observed phenotypes due to the SNP and Indel variants in the sitl1, Illumina-based RNA-seq was used to profile mRNA expression in root tissues. A set of 438 and 767 genes was identified whose mRNAs showed significantly increased or decreased transcript abundance respectively in the sitl1 (Figure S8 and Table S2-S3). The overrepresented gene functions of DEGs in the sitl1 were significantly associated with metal handling, stress, miscellaneous, protein, and transport functions (Figure S9). Analysis using metabolic pathways revealed that a large number of DEGs involved in a wide range of metabolisms including cell wall, light reactions, photosynthesis, and lipid metabolism were significantly downregulated in the sitl1 (Figure S10).

In an effort to evaluate whether the genes containing SNP and Indel variants in the sitl1 had altered gene expression levels, the mRNA expression profiles of 188 SNPs and 107 Indels variants were analyzed, which are commonly detected via WGS and RNA-seq. A total of 7 genes containing 4 SNP and 3 Indel variants showed significantly upregulated or downregulated expression levels in the sitl1 compared to WT (Figure 7c,d). Of these, four SNP variants were located in the upstream of Os05g0227600, downstream of Os01g0195400 and Os09g036770, or the 3' UTR region of Os08g0477100 (Figure 7c). In addition, two Indel variants were located in the upstream of Os08g0159500 and Os12g0226066 (Figure 7d). Lastly, one gene (Os01g0664100; MSU ID, Os04g47460, $\mathrm{Mg}^{2+}$ transporter, CorA-like $\mathrm{ZnB}$ domain) containing Indel variant in the CDS region has significantly downregulated mRNA abundance in the sitl1 (Figure 7d). This gene was selected and named (OsMTP1) as a putative causal gene for further functional analysis

\section{Mutation confirmation, subcellular localization, and expression patterns of OsMTP1}

In order to confirm the mutation in OsMTP1, genomic DNA was isolated and PCR-based molecular cloning was conducted. This was followed by Sanger DNA sequencing for analyzing the regions to see where the mutations predicted in gDNA of OsMTP1 were present. Consistent with the WGS analysis, we identified the insertion of T between nucleotide 4601 and 4062 in exon 5 of the mOsMTP1 gene (mutated Os01g47460 ) in the sitl1 (Figure 8a). Next, we explored whether this $\mathrm{T}$ insertion can lead to the incorrect splicing of the OsMTP1 mRNA in the sitl1. Sanger sequencing results showed that the T insertion was found between nucleotide 1044 and 1045 in mRNA of mOsMTP1 as expected but we did not find any other splicing forms of OsMTP1 (Figure 8a). The predicted protein sequence of mOsMTP1 in the sitl1 showed that this $\mathrm{T}$ insertion led to a frameshift in CorA-like ZntB cation transporter domain region of the mOsMTP1 protein sequence (LVSIILNQEIRRLATQVIRV to SSQHHIESRNQKVSNTGNQS, 349 - 368 amino acids) and the appearance of a premature stop codon (TAA) (Figure S11).

Gene expression levels of OsMTP1 were analyzed using two primer sets, which were designed in the Nor C- terminal region of theOsMTP1. Real-time qRT-PCR results with qRT1 primer set showed that the expression level of OsMTP1 was not changed between thesitl1 and WT (Figure 8b). However, gene expression levels of OsMTP1 in the sitl1 significantly lower than that of WT in both roots and leaves when we used qRT2 primer set (Figure 8c). One possible explanation for this is the premature termination codon containing aberrant mRNAs of the mOsMTP1 caused degradation due to eukaryotic quality control system (Mekawy et al., 2015; Nyiko et al., 2017). These results suggest that a single nucleotide insertion in codon 349 of themOsMTP1 gene might disrupt existing gene functions and gene expression of OsMTP1 in the sitl1 
OsMTP1 protein contains putative metal ion transporter CorA-like ZntB cation transporter domain with 2 transmembrane (TM) domains in the C-terminal regions. The alignment between OsMTP1 and its one paralog in rice (Os01g41720) and other orthologs from sorghum, maize, and Arabidopsis evidenced highly conserved the CorA-like ZntB magnesium transporter domain containing conserved GIN-motif (Figure S12 and S13). A phylogeny from the previous studies of MRS2/MGT gene family in rice and Arabidopsis (Lalonde et al., 2017) with the OsMTP1 and its orthologs showed a clear distinction between the MRS2/MGT and OsMTP1 with its orthologs, suggesting divergence in function (Figure S14). We also confirmed that subcellular localization of the 35S::OsMTP1-sGFP was clearly co-localized with the plasma membrane marker (pm-rk) in rice protoplasts (Figure 8d)

Lastly, we evaluated OsMTP1 expression patterns using various tissues from different developmental stages in WT rice (Figure 8e). The OsMTP1 was rarely expressed in leaf sheath compared to other tissues, whereas it was highly induced in root and leaf blade tissues at young seedling stage. The expression levels of OsMTP1 were gradually reduced in all tissues at late vegetative stage and the reproductive stage. Further examination of the expression levels ofOsMTP1 in response to salinity stress in 1-week-old root and leaf tissues was evaluated (Figure 8f-g). In roots, the OsMTP1 gene was upregulated at $30 \mathrm{~m}$ after $\mathrm{NaCl}$ treatment and highly induced with peak expression occurring at $1 \mathrm{~h}$. The induced mRNA abundances of theOsMTP1 were dramatically reduced at 6 to $24 \mathrm{~h} \mathrm{NaCl}$ treatment in roots (Figure 8f). Similar expression pattern of the OsMTP1 was observed in leaf tissues. The OsMTP1 exhibited increased transcript abundance at $30 \mathrm{~m}$ until $6 \mathrm{~h}$ by $\mathrm{NaCl}$ treatment and then reduced dramatically (Figure 8g). Collectively, these results showed that OsMTP1 mainly functions in roots and leaves in the early response to salinity stress and $\mathrm{T}$ insertion mutation in the OsMTP1 led to decrease in $\mathrm{Na}^{+}$and $\mathrm{Mg}^{2+}$ uptake at plasma membrane resulting in salinity tolerance in the sitl1 .

\section{Functional complementation and heterologous overexpression assay in yeast}

A complementation assay was performed using the yeast mutant CM66, which lacks two magnesium transporters (ALR1 and ALR2), and CM52 (wild-type) derived from FY833 (Saito et al., 2013). The mutant CM66 yeast cells cannot grow on $\mathrm{Mg}^{2+}$ concentrations of $<4 \mathrm{mM}$ in culture medium (Winston, Dollard, \& Ricupero-Hovasse, 1995). Heterogeneous expression of the OsMTP1 gene in CM66 showed this yeast stain was able to complement growth on both solid and liquid media supplanted with 0.1 and $1 \mathrm{mM} \mathrm{Mg} \mathrm{Mg}^{2+}$, whereas expression of the mOsMTP1 gene in CM66 did not alter yeast cell growth at lower levels of $\mathrm{Mg}^{2+}$ compared to the CM66 and EV (Figure 9a,b). This result indicates that heterogeneous expression of OsMTP1 is able to direct $\mathrm{Mg}^{2+}$ uptake into yeast.

The observed salinity insensitive phenotype and the reduced $\mathrm{Mg}^{2+}$ and $\mathrm{Na}^{+}$concentrations in plant tissues and in xylem sap in the sitl1 led us to hypothesize that the OsMTP1 could have ability to uptake not only $\mathrm{Mg}^{2+}$ but also $\mathrm{Na}^{+}$ion as a function of cotransporter activity (Figure 4-5). We next explored whether heterogeneous expression of the OsMTP1 in WT yeast can reduce cell growth on standard media containing $\mathrm{NaCl}$ due to increasing $\mathrm{Na}^{+}$uptake by OsMTP1 (Figure 9c-h). Indeed, heterologous expression of OsMTP1 showed reduced cell growth rate of yeast due to higher sensitivity to $\mathrm{NaCl}(0.5$ and $1 \mathrm{M})$ compared to control lines (Figure 9c-f). The OsMTP1 transformants showed no remarkable difference in their growth when medium containing $1 \mathrm{M} \mathrm{NaCl}$ along with $\mathrm{Mg}^{2+}$ at a low $0.1 \mathrm{mM}$ concentration (Figure $9 \mathrm{~g}$ ). However, this reduced cell growth of the OsMTP1 transformant could be rescued in the presence of $1 \mathrm{mM} \mathrm{Mg}^{2+}$ (Figure 9h). These results demonstrate that the OsMTP1 protein might play important roles to uptake both $\mathrm{Mg}^{2+}$ and $\mathrm{Na}^{+}$ions, and the higher concentrations of $\mathrm{Mg}^{2+}$ can compete with the rate of $\mathrm{Na}^{+}$influx at the plasma membrane.

\section{Discussion}

The ability of salinity tolerant plants to restrict the transporter and accumulations of $\mathrm{Na}^{+}$in leaf tissues and thereby avoid the effects of toxic $\mathrm{Na}^{+}$ion stress is among the most remarkable salt tolerance traits (L. Li et al., 2001). Plants have evolved physiological and biochemical mechanisms to adapt to salinity stress and many genes are involved in mediating the root-to-shoot translocation of $\mathrm{Na}^{+}$. In the present study, 
thesitl1 showed significantly improved tolerance to salinity stress due to reduced $\mathrm{Na}^{+}$uptake in roots and xylem sap (Figure 5). This reduced $\mathrm{Na}^{+}$concentrations might explain, in part, that relatively lower $\mathrm{Na}^{+}$ amount was translocated to aerial shoots of the sitl1, which results in decreased $\mathrm{H}_{2} \mathrm{O}_{2}$ accumulation and a salinity insensitive phenotype (Figure 4,5 ). In order to gather clues into the mechanistic basis for the observed salinity tolerance of thesitl1, we explored changes in relative mRNA abundances of marker genes involved in antioxidant enzyme genes, and $\mathrm{Na}^{+}$and $\mathrm{K}^{+}$transporter genes. The reduced $\mathrm{Na}^{+}$accumulation in both root and leaf tissues of thesitl1 also resulted in decreased mRNA abundances of most antioxidant enzyme genes under salinity stress condition (Figure 6). However, several genes showed significantly different expression levels between the sitl1 and WT under normal condition. For example, OsCAT1 showed decreased and increased mRNA abundances in roots and leaves, respectively. Furthermore, both $\mathrm{Na}^{+}$transporters, OsHKT1;5 and OsSOS1, showed increased mRNA abundance in root under normal condition. These results indicate that $\mathrm{Mg}+$ deficiency in the sitl1 might result in transcriptional reprograming, which led to changes mRNA abundance of these marker genes.

Salinity stress is known to induce changes in various essential ion elements uptakes such as N, P, K, Ca, Mg, and $\mathrm{Mn}$ in plants. In rice, several studies have reported that high level of $\mathrm{Na}^{+}$concentration in soils resulted in decreased ability to uptake $\mathrm{Mg}^{2+}$ due to the suppressive effect of $\mathrm{Na}^{+}$(Akter \& Oue, 2018; Hakim et al., 2014; Munns \& Tester, 2008). Similar trends are evident when the sitl1 and WT plants exposed to the salinity stress. As shown in Figure 5, content of $\mathrm{K}, \mathrm{Mg}$, and $\mathrm{Ca}$ in the roots and leaves were reduced under salinity stress condition. These results indicate that some transporters or channels of these cations might reduce their transport activity or co-transporters such as $\mathrm{Na}^{+} / \mathrm{Mg}^{2+}$ or $\mathrm{Na}^{+} / \mathrm{Ca}^{2+}$ symporters associated with this trend. To the best of our understanding, $\mathrm{Na}^{+}$and $\mathrm{Mg}^{2+}$ symporters have not been reported in organisms. However, there is an evidence that some transporter can transport both monovalent and divalent ions. For example, both TaHKT2;1 and OsHKT2;4 exhibited strong $\mathrm{K}^{+}$permeability in X. laevis oocytes ; however, TaHKT2;1 showed a small $\mathrm{Mg}^{2+}$ permeability and the OsHKT2;4 showed $\mathrm{Mg}^{2+}$ and $\mathrm{Ca}^{2+}$ permeability in the absence of competing $\mathrm{K}^{+}$ions, respectively (Munns \& Tester, 2008). In addition, $\mathrm{Na}^{+} / \mathrm{Mg}^{2+}$ transporter and $\mathrm{Na}^{+} / \mathrm{Ca}^{2+}$ exchanger (AtNCL) have been reported to play an important role in $\mathrm{Mg}^{2+}$ and $\mathrm{Ca}^{2+}$ homeostasis in human and plants, respectively (Horie et al., 2011; Wang et al., 2012). Another possibility is that extracellular $\mathrm{Mg}^{2+}$ level could directly alter $\mathrm{Na}^{+}$influx through non-selective cation channel (Sontia \& Touyz, 2007). Similarly, $\mathrm{Mg}^{2+}$ transporter, OsMGT1, which might enhance the transporter activity of OsHKT1;5 suggests decreased $\mathrm{Na}^{+}$accumulation to the shoots (Davenport \& Tester, 2000). In the present study, we identified OsMTP1 is possible causal mutation in the sitl1 via WGS and RNA-seq analyses (Figure 7). The sitl1 showed reduction of both $\mathrm{Na}^{+}$and $\mathrm{Mg}^{2+}$ ions in roots, leave and xylem sap (Figure 5). This significant reduction in $\mathrm{Mg}^{2+}$ amount in tissues resulted in reduced root growth and chlorophyll content in leaves under normal growth condition (Figure 1-3). However, the $\mathrm{Mg}^{2+}$ deficient symptoms of thesitill were restored to the level like that of WT when this mutant was grown under nutrient solution containing $500 \mu \mathrm{M}$ of $\mathrm{Mg}^{2+}$ (Figure S4). In addition, the sitl1 showed salinity insensitivity under both nutrient solution and DW containing $\mathrm{Na}^{+}(50 \mathrm{mM})$. Taken together, these results suggest that OsMTP1 is related to transport both ions, $\mathrm{Na}^{+}$and $\mathrm{Mg}^{2+}$ in plants, resulting in salinity insensitivity and $\mathrm{Mg}^{2+}$ deficiency in the sitl1 .

In the present study, we found a single nucleotide insertion in plasma membrane-localized OsMTP1 containing CorA-like ZntB type cation transporter domain in the sitl1 (Figure 8). Further analyses of gDNA and cDNA sequences confirmed that the mOsMTP1 may not be functional due to the appearance of a premature STOP codon between 1044 and 1045 inmOsMTP1 transcript. In rice, nine members of OsMRS2/MGT homologs of bacterial CorA-type $\mathrm{Mg}^{2+}$ transporter were isolated and examined their $\mathrm{Mg}^{2+}$ transport activity using a yeast complementation assay (Chen et al., 2017). The ability of $\mathrm{Mg}^{2+}$ transport activity was reported for four (OsMRS2-1, OsMRS2-3, OsMRS2-6 and OsMRS2-9) out of nine members have shown $\mathrm{Mg}^{2+}$ transport activity in yeast CM66. Also, OsMRS2-2 (OsMGT1) was identified to have $\mathrm{Mg}^{2+}$ transport ability in X. laevis oocytes and plants (Chen et al., 2017; Saito et al., 2013). Among them, OsMRS2-6 showed most effective cell growth rate in the complementation of the yeast CM66 in liquid medium containing $0.1 \mathrm{mM}$ $\mathrm{Mg}^{2+}$ indicating it to be a high-affinity $\mathrm{Mg}^{2+}$ transporter. In the present study, the yeast complementation 
assay of the OsMTP1 showed effective cell growth rate which suggested that OsMTP1 functions as $\mathrm{Mg}^{2+}$ transporter (Figure 9a). A phylogenetic tree analysis showed that the OsMTP1 protein was clearly distinct from Arabidopsis and rice MRS2 family; however, the OsMRS2-6 is the most closely related member of the OsMTP1 which shares the ability of $\mathrm{Mg}^{2+}$ transport (Figure S10). The ionomic analysis of the sitl1 showed the reduced $\mathrm{Mg}^{2+}$ content but also reduced $\mathrm{Na}^{+}$which led us to test the ability of $\mathrm{Na}^{+}$transport. Heterogeneous expression of OsMTP1 showed increased salinity sensitivity based on yeast cell growth however this salinity sensitivity was affected by $\mathrm{Mg}^{2+}$ concentration (Figure 9c-h). As shown in Figure 9, increased concentration of $\mathrm{Mg}^{2+}$ in complete media markedly reduced $\mathrm{Na}^{+}$senility in yeasts. In addition, thesitl1 showed salinity stress tolerance under deionized water containing $\mathrm{NaCl}$ supplemented with no $\mathrm{Mg}^{2+}$ condition which indicated that the OsMTP1 is capable to transport $\mathrm{Na}^{+}$ions but the $\mathrm{Na}^{+}$transport activity might be affected by $\mathrm{Mg}^{2+}$ concentration.

The characteristic GMN motif located at the end of the first TM domain is conserved in most members of AtMRS2 and OsMRS2, whereas OsMTP1 has altered GIN tripeptide motif (Figure S13). The conserved GMN motif is suggested to be essential to $\mathrm{Mg}^{2+}$ transport ability, thus if the glycine residue of the GMN motif is substituted by alanine, the $\mathrm{Mg}^{2+}$ transport activity of yeast MRS2 transporter was abolished (Chen, Yamaji, Motoyama, Nagamura, \& Ma, 2012; Knoop et al., 2005). Indeed, this conserved GMN motif was shared by five members of OsMRS2-1, OsMRS2-2, OsMRS2-3, OsMRS2-6 and OsMRS2-9 which confirmed their $\mathrm{Mg}^{2+}$ transport activity in yeast and rice. However, further evidence for the importance of functional diversity was reported in CorA family. For instance, TmCorA in T. maritina with the GMN motif has been reported to play a role in cobalt transport with no $\mathrm{Mg}^{2+}$ transport activity (Kolisek et al., 2003) and also OsMRS2-7 with GMN motif failed to show the $\mathrm{Mg}^{2+}$ transport activity in the yeast complementation assay (Xia et al., 2011). The OsMTP1 and its orthologs in Gramineae contains well-conserved GIN motif in CorA-like ZntB cation transport domain which was reported to associate with $\mathrm{Zn}^{2+}$ and $\mathrm{Cd}^{2+}$ selectivity in $S$. typhimurium. Subcellular localization, yeast complementation, and inomic analyses suggest that a plasma membrane localized OsMTP1 harboring CorA-like ZntB cation transfer domain might have important roles in regulating both $\mathrm{Na}^{+}$and $\mathrm{Mg}^{2+}$ homeostasis in rice. However, further studies are required to decipher its exact role.

\section{Author contributions}

CSJ conceived of the overall study. SDL, JHK, JL and SGH conducted experiments and analyzed the data. SDL, SGH, and CSJ co-wrote the paper. All authors have read and approved the final manuscript.

\section{Declaration of Competing Interest}

The authors declare that the research was conducted in the absence of any commercial or financial relationships that could be construed as a potential conflict of interest.

\section{Acknowledgements}

This work was supported by a grant from "Cooperative Research Program for Agriculture Science and Technology Department (Project No. PJ013429012019)", Rural Development Administration, Republic of Korea. S.D.L. acknowledges support from the Basic Science Research Program through the National Research Foundation of Korea (NRF) funded by the Ministry of Education (NRF-2019R1I1A1A01061727). The authors would like to thank to professor Keitaro Tanoi for providing CM66 and CM52 yeast cell lines. Special thanks to Dr. Pradeep Yerramsetty for providing clarifying comments on the manuscript.

\section{References}

Akter, M., \& Oue, H. (2018). Effect of Saline Irrigation on Accumulation of Na+, K+, Ca2+, and Mg2+ Ions in Rice Plants.Agriculture-Basel, 8 (10). Retrieved from < Go to ISI>://WOS:000448539000019

Almeida, D. M., Oliveira, M. M., \& Saibo, N. J. M. (2017). Regulation of Na+ and K+ homeostasis in plants: towards improved salt stress tolerance in crop plants. Genetics and Molecular Biology, 40 (1 suppl 1), 326-345. doi:10.1590/1678-4685-GMB-2016-0106 
Apse, M. P., Aharon, G. S., Snedden, W. A., \& Blumwald, E. (1999). Salt tolerance conferred by overexpression of a vacuolar Na+/H+ antiport in Arabidopsis. Science, 285 (5431), 1256-1258. doi:10.1126/science.285.5431.1256

Arnon, D. I. (1949). Copper Enzymes in Isolated Chloroplasts. Polyphenoloxidase in Beta Vulgaris. Plant Physiology, 24 (1), 1-15. doi:10.1104/pp.24.1.1

Berthomieu, P., Conejero, G., Nublat, A., Brackenbury, W. J., Lambert, C., Savio, C., . . . Casse, F. (2003). Functional analysis of AtHKT1 in Arabidopsis shows that $\mathrm{Na}(+)$ recirculation by the phloem is crucial for salt tolerance. EMBO Journal, 22 (9), 2004-2014. doi:10.1093/emboj/cdg207

Bi, Y. M., Kant, S., Clarke, J., Gidda, S., Ming, F., Xu, J., . . . Rothstein, S. J. (2009). Increased nitrogen-use efficiency in transgenic rice plants over-expressing a nitrogen-responsive early nodulin gene identified from rice expression profiling. Plant Cell $\&$ Environment, 32 (12), 1749-1760. doi:10.1111/j.13653040.2009.02032.x

Byrt, C. S., Platten, J. D., Spielmeyer, W., James, R. A., Lagudah, E. S., Dennis, E. S., . . . Munns, R. (2007). HKT1;5-like cation transporters linked to Na+ exclusion loci in wheat, Nax2 and Kna1.Plant Physiology, 143 (4), 1918-1928. doi:10.1104/pp.106.093476

Chen, Z. C., Yamaji, N., Horie, T., Che, J., Li, J., An, G., \& Ma, J. F. (2017). A Magnesium Transporter OsMGT1 Plays a Critical Role in Salt Tolerance in Rice. Plant Physiology, 174 (3), 1837-1849. doi:10.1104/pp.17.00532

Chen, Z. C., Yamaji, N., Motoyama, R., Nagamura, Y., \& Ma, J. F. (2012). Up-regulation of a magnesium transporter gene OsMGT1 is required for conferring aluminum tolerance in rice. Plant Physiology, 159 (4), 1624-1633. doi:10.1104/pp.112.199778

Chinnusamy, V., Jagendorf, A., \& Zhu, J. K. (2005). Understanding and improving salt tolerance in plants. Crop Science, 45 (2), 437-448. doi:DOI 10.2135/cropsci2005.0437

Cho, H. Y., Park, S. J., Kim, D. S., \& Jang, C. S. (2010). A TILLING rice population induced by gamma-ray irradiation and its genetic diversity. Korean Journal of Breeding Science, 42 (4), 365-373.

Cotsaftis, O., Plett, D., Shirley, N., Tester, M., \& Hrmova, M. (2012). A two-staged model of Na+ exclusion in rice explained by 3D modeling of HKT transporters and alternative splicing. PLoS One, 7 (7), e39865. doi:10.1371/journal.pone.0039865

Daudi, A., \& O'Brien, J. A. (2012). Detection of Hydrogen Peroxide by DAB Staining in Arabidopsis Leaves. Bio-Protocol, 2 (18), e263. Retrieved from https://www.ncbi.nlm.nih.gov/pubmed/27390754

Davenport, R. J., \& Tester, M. (2000). A weakly voltage-dependent, nonselective cation channel mediates toxic sodium influx in wheat.Plant Physiology, 122 (3), 823-834. doi:10.1104/pp.122.3.823

Ding, L., \& Zhu, J. K. (1997). Reduced Na+ uptake in the NaCl-hypersensitive sos1 mutant of Arabidopsis thaliana. Plant Physiology, 113 (3), 795-799. doi:10.1104/pp.113.3.795

Fukuda, A., Nakamura, A., Hara, N., Toki, S., \& Tanaka, Y. (2011). Molecular and functional analyses of rice NHX-type Na+/H+ antiporter genes. Planta, 233 (1), 175-188. doi:10.1007/s00425-010-1289-4

Fukuda, A., Nakamura, A., Tagiri, A., Tanaka, H., Miyao, A., Hirochika, H., \& Tanaka, Y. (2004). Function, intracellular localization and the importance in salt tolerance of a vacuolar $\mathrm{Na}(+) / \mathrm{H}(+)$ antiporter from rice. Plant 83 Cell Physiology, 45 (2), 146-159. doi:10.1093/pcp/pch014

Gay, C., \& Gebicki, J. M. (2000). A critical evaluation of the effect of sorbitol on the ferric-xylenol orange hydroperoxide assay.Analytical Biochemistry, 284 (2), 217-220. doi:10.1006/abio.2000.4696

Hacham, Y., Holland, N., Butterfield, C., Ubeda-Tomas, S., Bennett, M. J., Chory, J., \& Savaldi-Goldstein, S. (2011). Brassinosteroid perception in the epidermis controls root meristem size.Development, 138 (5), 839-848. doi:10.1242/dev.061804 
Hakim, M. A., Juraimi, A. S., Hanafi, M. M., Ismail, M. R., Rafii, M. Y., Islam, M. M., \& Selamat, A. (2014). The Effect of Salinity on Growth, Ion Accumulation and Yield of Rice Varieties. Journal of Animal and Plant Sciences, 24 (3), 874-885. Retrieved from < Go to ISI >://WOS:000339234900030

Hermans, C., \& Verbruggen, N. (2005). Physiological characterization of Mg deficiency in Arabidopsis thaliana. Journal of Experimental Botany, 56 (418), 2153-2161. Retrieved from < Go to ISI >://WOS:000230513500017

Hermans, C., Vuylsteke, M., Coppens, F., Cristescu, S. M., Harren, F. J. M., Inze, D., \& Verbruggen, N. (2010). Systems analysis of the responses to long-term magnesium deficiency and restoration in Arabidopsis thaliana. New Phytologist, 187 (1), 132-144. Retrieved from < Go to ISI>://WOS:000278395100014

Horie, T., Brodsky, D. E., Costa, A., Kaneko, T., Lo Schiavo, F., Katsuhara, M., \& Schroeder, J. I. (2011). K+ Transport by the OsHKT2;4 Transporter from Rice with Atypical Na+ Transport Properties and Competition in Permeation of K+ over Mg2+ and Ca2+ Ions. Plant Physiology, 156 (3), 1493-1507. doi:10.1104/pp.110.168047

Horie, T., Costa, A., Kim, T. H., Han, M. J., Horie, R., Leung, H. Y., . . . Schroeder, J. I. (2007). Rice OsHKT2;1 transporter mediates large $\mathrm{Na}+$ influx component into $\mathrm{K}+$-starved roots for growth. EMBO Journal, 26 (12), 3003-3014. doi:10.1038/sj.emboj.7601732

Huang, S. B., Spielmeyer, W., Lagudah, E. S., James, R. A., Platten, J. D., Dennis, E. S., \& Munns, R. (2006). A sodium transporter (HKT7) is a candidate for Nax1, a gene for salt tolerance in durum wheat.Plant Physiology, 142 (4), 1718-1727. doi:10.1104/pp.106.088864

Hwang, S. G., Hwang, J. G., Kim, D. S., \& Jang, C. S. (2014). Genome-wide DNA polymorphism and transcriptome analysis of an early-maturing rice mutant. Genetica, 142 (1), 73-85. doi:10.1007/s10709-0139755-0

Islam, F., Farooq, M. A., Gill, R. A., Wang, J., Yang, C., Ali, B., . . . Zhou, W. (2017). 2,4-D attenuates salinity-induced toxicity by mediating anatomical changes, antioxidant capacity and cation transporters in the roots of rice cultivars. Sci Rep, 7 (1), 10443. doi:10.1038/s41598-017-09708-x

Jabnoune, M., Espeout, S., Mieulet, D., Fizames, C., Verdeil, J. L., Conejero, G., . . . Very, A. A. (2009). Diversity in expression patterns and functional properties in the rice HKT transporter family.Plant Physiology, 150 (4), 1955-1971. doi:10.1104/pp.109.138008

Knoop, V., Groth-Malonek, M., Gebert, M., Eifler, K., \& Weyand, K. (2005). Transport of magnesium and other divalent cations: evolution of the 2-TM-GxN proteins in the MIT superfamily. Molecular Genetics and Genomics, 274 (3), 205-216. doi:10.1007/s00438-005-0011-x

Kolisek, M., Zsurka, G., Samaj, J., Weghuber, J., Schweyen, R. J., \& Schweigel, M. (2003). Mrs2p is an essential component of the major electrophoretic Mg2+ influx system in mitochondria. EMBO Journal, 22 (6), 1235-1244. doi:10.1093/emboj/cdg122

Krishnamurthy, P., Ranathunge, K., Nayak, S., Schreiber, L., \& Mathew, M. K. (2011). Root apoplastic barriers block Na+ transport to shoots in rice (Oryza sativa L.). Journal of Experimental Botany, 62 (12), 4215-4228. doi:10.1093/jxb/err135

Lalonde, S., Stone, O. A., Lessard, S., Lavertu, A., Desjardins, J., Beaudoin, M., . . . Lettre, G. (2017). Frameshift indels introduced by genome editing can lead to in-frame exon skipping. PLoS One, 12 (6), e0178700. doi:10.1371/journal.pone.0178700

Li, H., \& Durbin, R. (2009). Fast and accurate short read alignment with Burrows-Wheeler transform. Bioinformatics, 25 (14), 1754-1760. doi:10.1093/bioinformatics/btp324

Li, L., Tutone, A. F., Drummond, R. S., Gardner, R. C., \& Luan, S. (2001). A novel family of magnesium transport genes in Arabidopsis.Plant Cell, 13 (12), 2761-2775. doi:10.1105/tpc.010352 
Lim, S. D., Kim, S. H., Gilroy, S., Cushman, J. C., \& Choi, W. G. (2019). Quantitative ROS bioreporters: A robust toolkit for studying biological roles of ROS in response to abiotic and biotic stresses. Physiologia Plantarum, 165 (2), 356-368. doi:10.1111/ppl.12866

Lim, S. D., Lee, C., \& Jang, C. S. (2014). The rice RING E3 ligase, OsCTR1, inhibits trafficking to the chloroplasts of OsCP12 and OsRP1, and its overexpression confers drought tolerance in Arabidopsis. Plant Cell E Environment, 37 (5), 1097-1113. doi:10.1111/pce.12219

Lim, S. D., Yim, W. C., Liu, D., Hu, R., Yang, X., \& Cushman, J. C. (2018). A Vitis vinifera basic helix-loop-helix transcription factor enhances plant cell size, vegetative biomass and reproductive yield.Plant Biotechnology Journal, 16 , 1595-1615. doi:10.1111/pbi.12898

Livak, K. J., \& Schmittgen, T. D. (2001). Analysis of relative gene expression data using real-time quantitative PCR and the 2(-Delta Delta C(T)) Method. Methods, 25 (4), 402-408. doi:10.1006/meth.2001.1262

Ma, J. F., Goto, S., Tamai, K., \& Ichii, M. (2001). Role of root hairs and lateral roots in silicon uptake by rice. Plant Physiology, 127 (4), 1773-1780. Retrieved from https://www.ncbi.nlm.nih.gov/pubmed/11743120

Martinez-Atienza, J., Jiang, X., Garciadeblas, B., Mendoza, I., Zhu, J. K., Pardo, J. M., \& Quintero, F. J. (2007). Conservation of the salt overly sensitive pathway in rice. Plant Physiology, 143 (2), 1001-1012. doi:10.1104/pp.106.092635

Maser, P., Hosoo, Y., Goshima, S., Horie, T., Eckelman, B., Yamada, K., . . . Uozumi, N. (2002). Glycine residues in potassium channel-like selectivity filters determine potassium selectivity in four-loop-per-subunit HKT transporters from plants. Proc Natl Acad Sci U S A, 99 (9), 6428-6433. doi:10.1073/pnas.082123799

Meier, S. D., Kovalchuk, Y., \& Rose, C. R. (2006). Properties of the new fluorescent Na+ indicator CoroNa Green: comparison with SBFI and confocal Na+ imaging. Journal of Neuroscience Methods, 155 (2), 251-259. doi:10.1016/j.jneumeth.2006.01.009

Mekawy, A. M., Assaha, D. V., Yahagi, H., Tada, Y., Ueda, A., \& Saneoka, H. (2015). Growth, physiological adaptation, and gene expression analysis of two Egyptian rice cultivars under salt stress.Plant Physiology and Biochemistry, 87, 17-25. doi:10.1016/j.plaphy.2014.12.007

Mian, A., Oomen, R. J., Isayenkov, S., Sentenac, H., Maathuis, F. J., \& Very, A. A. (2011). Over-expression of an Na+-and K+-permeable HKT transporter in barley improves salt tolerance. Plant Journal, 68 (3), 468-479. doi:10.1111/j.1365-313X.2011.04701.x

Munns, R. (2005). Genes and salt tolerance: bringing them together.New Phytologist, 167 (3), 645-663. doi:10.1111/j.1469-8137.2005.01487.x

Munns, R., \& Tester, M. (2008). Mechanisms of salinity tolerance.Annual Review of Plant Biology, 59 , 651-681. doi:10.1146/annurev.arplant.59.032607.092911

Nakagawa, T., Suzuki, T., Murata, S., Nakamura, S., Hino, T., Maeo, K., . . . Ishiguro, S. (2007). Improved Gateway binary vectors: high-performance vectors for creation of fusion constructs in transgenic analysis of plants. Biosci Biotechnol Biochem, 71 (8), 2095-2100. doi:10.1271/bbb.70216

Nelson, B. K., Cai, X., \& Nebenfuhr, A. (2007). A multicolored set of in vivo organelle markers for colocalization studies in Arabidopsis and other plants. Plant Journal, 51 (6), 1126-1136. doi:10.1111/j.1365313X.2007.03212.x

Nyiko, T., Auber, A., Szabadkai, L., Benkovics, A., Auth, M., Merai, Z., . . . Silhavy, D. (2017). Expression of the eRF1 translation termination factor is controlled by an autoregulatory circuit involving readthrough and nonsense-mediated decay in plants. Nucleic Acids Research, 45 (7), 4174-4188. doi:10.1093/nar/gkw1303

Park, M., Lee, H., Lee, J. S., Byun, M. O., \& Kim, B. G. (2009). In Planta Measurements of Na+ Using Fluorescent Dye CoroNa Green.Journal of Plant Biology, 52 (4), 298-302. doi:10.1007/s12374-009-9036-8 
Patel, R. K., \& Jain, M. (2012). NGS QC Toolkit: a toolkit for quality control of next generation sequencing data. PLoS One, 7 (2), e30619. doi:10.1371/journal.pone.0030619

Platten, J. D., Cotsaftis, O., Berthomieu, P., Bohnert, H., Davenport, R. J., Fairbairn, D. J., . . . Tester, M. (2006). Nomenclature for HKT transporters, key determinants of plant salinity tolerance. Trends in Plant Science, 11 (8), 372-374. doi:10.1016/j.tplants.2006.06.001

Ren, Z. H., Gao, J. P., Li, L. G., Cai, X. L., Huang, W., Chao, D. Y., . . . Lin, H. X. (2005). A rice quantitative trait locus for salt tolerance encodes a sodium transporter. Nature Genetics, 37 (10), 1141-1146. doi:10.1038/ng1643

Saito, T., Kobayashi, N. I., Tanoi, K., Iwata, N., Suzuki, H., Iwata, R., \& Nakanishi, T. M. (2013). Expression and functional analysis of the CorA-MRS2-ALR-type magnesium transporter family in rice. Plant $\&$ Cell Physiology, 54 (10), 1673-1683. doi:10.1093/pcp/pct112

Schachtman, D. P., \& Schroeder, J. I. (1994). Structure and transport mechanism of a high-affinity potassium uptake transporter from higher plants. Nature, 370 (6491), 655-658. doi:10.1038/370655a0

Schock, I., Gregan, J., Steinhauser, S., Schweyen, R., Brennicke, A., \& Knoop, V. (2000). A member of a novel Arabidopsis thaliana gene family of candidate $\mathrm{Mg} 2+$ ion transporters complements a yeast mitochondrial group II intron-splicing mutant. Plant Journal, 24 (4), 489-501. doi:10.1046/j.1365-313x.2000.00895.x

Shaul, O. (2002). Magnesium transport and function in plants: the tip of the iceberg. BioMetals, 15 (3), 309-323. doi:10.1023/a:1016091118585

Shi, H., Ishitani, M., Kim, C., \& Zhu, J. K. (2000). The Arabidopsis thaliana salt tolerance gene SOS1 encodes a putative $\mathrm{Na}+\mathrm{H}+$ antiporter.Proc Natl Acad Sci U S A, 97 (12), 6896-6901. doi:10.1073/pnas.120170197

Shi, H., Quintero, F. J., Pardo, J. M., \& Zhu, J. K. (2002). The putative plasma membrane Na(+)/H(+) antiporter SOS1 controls long-distance $\mathrm{Na}(+)$ transport in plants. Plant Cell, 14 (2), 465-477. doi:10.1105/tpc.010371

Sontia, B., \& Touyz, R. M. (2007). Magnesium transport in hypertension.Pathophysiology, 14 (3-4), 205-211. doi:10.1016/j.pathophys.2007.09.005

Sunarpi, Horie, T., Motoda, J., Kubo, M., Yang, H., Yoda, K., . . . Uozumi, N. (2005). Enhanced salt tolerance mediated by AtHKT1 transporter-induced Na unloading from xylem vessels to xylem parenchyma cells. Plant Journal, 44 (6), 928-938. doi:10.1111/j.1365-313X.2005.02595.x

Talei, D., Kadir, M. A., Yusop, M. K., Valdiani, A., \& Abdullah, M. P. (2012). Salinity effects on macro and micronutrients uptake in medicinal plant King of Bitters (Andrographis paniculata Nees.). Plant Omics, 5 (3), 271-278. Retrieved from < Go to ISI >://WOS:000310985300011

Walia, H., Wilson, C., Condamine, P., Liu, X., Ismail, A. M., Zeng, L., . . . Close, T. J. (2005). Comparative transcriptional profiling of two contrasting rice genotypes under salinity stress during the vegetative growth stage. Plant Physiology, 139 (2), 822-835. doi:10.1104/pp.105.065961

Wang, P., Li, Z., Wei, J., Zhao, Z., Sun, D., \& Cui, S. (2012). A Na+/Ca2+ exchanger-like protein (AtNCL) involved in salt stress in Arabidopsis. Journal of Biological Chemistry, 287 (53), 44062-44070. doi:10.1074/jbc.M112.351643

Winston, F., Dollard, C., \& Ricupero-Hovasse, S. L. (1995). Construction of a set of convenient Saccharomyces cerevisiae strains that are isogenic to S288C. Yeast, 11 (1), 53-55. doi:10.1002/yea.320110107

Worlock, A. J., \& Smith, R. L. (2002). ZntB is a novel Zn2+ transporter in Salmonella enterica serovar Typhimurium. Journal of Bacteriology, 184 (16), 4369-4373. doi:10.1128/jb.184.16.4369-4373.2002

Xia, Y., Lundback, A. K., Sahaf, N., Nordlund, G., Brzezinski, P., \& Eshaghi, S. (2011). Co2+ selectivity of Thermotoga maritima CorA and its inability to regulate $\mathrm{Mg} 2+$ homeostasis present a new class of CorA proteins. Journal of Biological Chemistry, 286 (18), 16525-16532. doi:10.1074/jbc.M111.222166 
Yao, X., Horie, T., Xue, S., Leung, H. Y., Katsuhara, M., Brodsky, D. E., . . . Schroeder, J. I. (2010). Differential sodium and potassium transport selectivities of the rice OsHKT2;1 and OsHKT2;2 transporters in plant cells. Plant Physiology, 152 (1), 341-355. doi:10.1104/pp.109.145722

Yildirim, E., Karlidag, H., \& Turan, M. (2009). Mitigation of salt stress in strawberry by foliar K, $\mathrm{Ca}$ and $\mathrm{Mg}$ nutrient supply. Plant Soil and Environment, 55 (5), 213-221. Retrieved from <Go to ISI >://WOS:000267192300006

Zhang, Q., Lin, F., Mao, T., Nie, J., Yan, M., Yuan, M., \& Zhang, W. (2012). Phosphatidic acid regulates microtubule organization by interacting with MAP65-1 in response to salt stress in Arabidopsis.Plant Cell, 24 (11), 4555-4576. doi:10.1105/tpc.112.104182

Zhou, Q. Y., Wang, L., Cai, X., Wang, D., Hua, X. J., Qu, L. Q., . . . Chen, T. (2011). Net sodium fluxes change significantly at anatomically distinct root zones of rice (Oryza sativa L.) seedlings. Journal of Plant Physiology, 168 (11), 1249-1255. doi:10.1016/j.jplph.2011.01.017

\section{Figure legends}

Figure 1. sitl1 mutant decreases root growth and leaf chlorophyll content. Rice seeds of the sitl1 mutant and wild-type (WT) control were germinated and grown in half-strength KimuraB nutrient solution or deionized water for 3 weeks (a) Representative seedling images of the sitl1 mutant and WT plants at 2, 4, and 7 days after germination (DAG) and 3 weeks after germination (WAG) under nutrient solution condition. (b) Comparison of lengths of root, coleoptile, leaf sheath, and leaf blade ( $n=30$ with 3 replicates). (c) Fresh weight ( $n=30$ with 3 replicates). (d) Representative leaf images of the sitl1 mutant and WT plants at 7 DAG and 3 WAG. (e) Comparison of leaf chlorophyll content ( $n=6$ replicates). Leaves were sampled and measured total chlorophyll, chlorophyll A, and chlorophyll B at 7 DAG and 3 WAG. (f) Representative seedling images of the sitl1 mutant and WT plant at 7 DAG under deionized water condition. Seeds of the sitl1 mutant and WT were germinated and grown in deionized water for 7 days. (g) Comparison of lengths of root, leaf sheath, and leaf blade at 7 DAG ( $n=30$ with 3 replicates). (h) Fresh weight $(n=$ 30 with 3 replicates). (i) Leaf chlorophyll content $(n=6$ replicates). Value represent means $\pm \mathrm{SD}$, ns $=$ non-significant, ${ }^{*} p<0.05$ and ${ }^{* * *} p<0.001$, two-way ANOva with Sidak's multiple comparison test.

Figure 2. Analysis of the sitl1 mutant in root development. Rice seeds of the sitl1 mutant and wild-type (WT) control were germinated and grown in half-strength KimuraB nutrient solution (NS) or deionized water (DW) for 1 week. Detached roots were used to scan and measure for root development. (a) Representative images of 1-week-old stil1 mutant and WT roots. (b) Comparison of lateral root number in primary roots $(n$ $=30$ with 3 replicates). (c) Lateral root density ( $n=30$ with 3 replicates). (d) Average length of lateral root ( $n=30$ with 3 replicates). (e) Sum of lateral root length ( $n=30$ with 3 replicates). Lateral root number, average length of lateral root, and sum of lateral root length were analyzed within 3-cm root samples from root base. (f) Length of root immature zone ( $n=30$ with 3 replicates). Root immature zone was defined as the zone of the root where lateral roots were not detected from the root tip. (g) Length of root mature zone ( $n=30$ with 3 replicates). Root mature zone was defined as the zone of the root where lateral root initiation and development takes places to root base. (h) Length of primary root (PR) width $(n=30$ with 3 replicates). (i) Length of lateral root width ( $n=30$ with 3 replicates). Value represent means $+-\mathrm{SD}$, ns $=$ non-significant, ${ }^{* * *} p<0.001$, two-way ANOVA with Sidak's multiple comparison test.

Figure 3. sitl1 mutant decreases cell number and size in root tissue. Rice seeds of the sitl1 mutant and wild-type (WT) were germinated and grown in half-strength KimuraB solution for 1 week. One-weekold roots were detached and stained with propidium iodide (PI) solution for $10 \mathrm{~min}$. Root images were captured via confocal laser-scanning microscopy and the epidermal cells were used to measure cell size and number. (a) Representative images of root meristems of thesitl1 mutant and WT. The root epidermal cells are outlined with solid lines. Green, red, and blue lines with double arrowheads represent the lengths of the apical meristem, the basal meristem and the elongation/differentiation zone, respectively (Hacham et al., 2011; Lim et al., 2018). QC indicates the quiescent center. Scale bar, 40um. Quantification of (b) average cell number in the apical meristem, (c) cell number in the basal meristem and (d) total cell number in the 
meristem zone ( $n=9$ with 3 replicates). Quantification of (e) apical meristem length, (f) basal meristem length and $(\mathrm{g})$ total meristem length $(n=9$ with 3 replicates). Quantification of $(\mathrm{h})$ average cell length and (i) cell width in the apical and basal meristems ( $n=9$ with 3 replicates). (j) Representative images of cortical cells in mature root zone of the sitl1 mutant and WT. Quantification of average (k) cortical cell length, (l) cell width and $(\mathrm{m})$ cell area in the mature root zone ( $n=9$ with 3 replicates). Value represent means $\pm \mathrm{SD}$, ns $=$ non-significant, ${ }^{*} p<0.05,{ }^{* *} p<0.05$ and ${ }^{* * *} p<0.001$, Student's $t$-test (b, c, d, e, f, $\mathrm{g}, \mathrm{k}, \mathrm{l}$, and $\mathrm{m}$ ) and two-way ANOva with Sidak's multiple comparison test (h and i).

Figure 4. sitl1 mutant enhances salinity insensitivity by reducing Na+ influx across the plasma membrane. Rice seeds of thesitl1 mutant and wild-type (WT) were grown for 1 week under half-strength KimuraB nutrient solution (NS) or deionized water (DW) conditions. Seedlings were then treated with NS or DW containing $0,50,100 \mathrm{mM} \mathrm{NaCl}$ for 1 week. (a) Representative images of the sitl1 mutant and WT at 7 days after salinity treatment. Boxes with broken lines indicate the third leaf of the sitl1 mutant and WT. Scale bar, $5 \mathrm{~cm}$. Quantification of fresh weights of root, leaf sheath, and leaf blade tissues under (b) NS condition and (c) DW condition ( $n=30$ with 3 replicates). Quantification of leaf chlorophyll content under (d) NS condition and (e) condition ( $n=30$ with 3 replicates). (f) Representative DAB staining images of leaf blades of the sitl1 mutant and WT. Rice seeds of the sitl1 mutant WT were germinated and grown in half-strength NS for 1 week. Seedlings were then treated with NS containing 0,50,100 $\mathrm{mM} \mathrm{NaCl}$ for 1 week and leaf blades were stained with DAB solution to assess the accumulation of $\mathrm{H}_{2} \mathrm{O}_{2}$. Scale bar, $1 \mathrm{~mm}$. (g) Quantification of $\mathrm{H}_{2} \mathrm{O}_{2}$ content in root tissues ( $n=6$ with 3 replicates). (h) Quantification of $\mathrm{H}_{2} \mathrm{O}_{2}$ content in leaf blade tissues ( $n=6$ with 3 replicates). (i) Representative images of $\mathrm{Na}^{+}$accumulations in lateral roots of the sitl1 mutant and WT. One-week-old seedlings of the sitl1 mutant and WT were treated with NS containing $50 \mathrm{mM} \mathrm{NaCl}$ for $3 \mathrm{~h}$. The lateral roots were detached and stained with CoroNa-green AM and FM4-64 to visualize the accumulations of $\mathrm{Na}^{+}$in the vacuole. (j) Representative images of $\mathrm{Na}+$ distribution in rice protoplasts of thesitl1 mutant and WT. Leaf protoplasts were isolated and treated with 0 and 50 $\mathrm{mM} \mathrm{NaCl}$ solution for $1 \mathrm{~h}$. The CoroNa-green AM was used to visualize $\mathrm{Na}^{+}$distribution in protoplasts. (k) Quantification of CoroNa green intensity $(n=3$ replicates with average intensity of 50 protoplasts per replicate). Value represent means $+-\mathrm{SD}, \mathrm{ns}=$ non-significant, ${ }^{*} p<0.05$ and ${ }^{* * *} p<0.001$, two-way ANOvA with Sidak's multiple comparison test.

Figure 5. sitl1 mutant reduces the concentration of $\mathrm{Mg}^{2+}$ and $\mathrm{Na}^{+}$in root and leaf tissues. One-week-old rice seedlings of the sitl1 mutant and wild-type (WT) were treated with 0 or $50 \mathrm{mM} \mathrm{NaCl}$ solution for 1 week. Oven-dried root and leaf samples of the sitl1 mutant and WT were used to determine the concentrations of inorganic ions via ICP-OES ( $n=3$ replicates). (a) Potassium. (b) Magnesium. (c) Sodium. (d) Calcium. (e) Phosphorus. Xylem sap was collected from 1-week-old rice seedlings of the sitl1 mutant and WT after 0 or $50 \mathrm{mM} \mathrm{NaCl}$ treatments. Collected sap was used to determine the concentrations of inorganic ions via ICPOES ( $n=6$ replicates). Quantification of (f) Magnesium, (g) Sodium, and (h) Potassium concentrations in xylem sap. Value represent means $+-\mathrm{SD}$, ns $=$ non-significant, ${ }^{*} p<0.05$ and ${ }^{* * *} p<0.001$, two-way ${ }_{\text {ANOVA }}$ with Sidak's multiple comparison test.

Figure 6. Relative fold expression of the genes encoding antioxidant defense enzymes, $\mathrm{Na}^{+}$, and $\mathrm{K}^{+}$transporters. One-week-old seedlings of the sitl1 mutant and WT were treated with half-strength KimuraB solution containing 0 or $50 \mathrm{mM} \mathrm{NaCl}$ for $1 \mathrm{~h}$. Relative expression levels of selected marker genes in root and leaf tissues were determined by qRT-PCR. (A) Relative expression of the genes encoding antioxidant defense enzymes (OsCAT1, Catalase isozyme A;OsCAT2, Catalase isozyme B; OsAPX1, Cytosolic ascorbate peroxidase 1; OsAPX2, Cytosolic ascorbate peroxidase 2;OsCuZnSOD1, Cytosolic copper/zinc-superoxide dismutase 1; OsMnSOD , Mitochondrial manganese-superoxide dismutase; OsPOD , Peroxidase; OsGR1, Cytosolic glutathione reductase 1; OsGR2, Mitochondrial glutathione reductase; OsDHAR1, Dehydroascorbate reductase; OsMDHAR1, Cytosolic monodehydroascorbate reductase; OsMDHAR2 , Putative monodehydroascorbate reductase; OsP5CS, Delta-1-pyrroline-5-carboxylate synthase). (B) Relative expression of the genes encoding $\mathrm{Na}^{+}$and $\mathrm{K}^{+}$transporters (OsHKT1;5, Sodium transporter Hkt1.5;OsLti6a, Plasma membrane protein 3 homolog; OsLti6b , Plasma membrane protein 3 homolog; OsHKT2;1, High-affinity potassium transporter; OsNHX1, Vacuolar $\mathrm{Na}^{+} / \mathrm{H}^{+}$antiporter; OsSOS1, Salt overly sensitive 1; OsAKT1 
, AKT-type $\mathrm{K}^{+}$channels; $H A K^{7}$, Potassium transporter 7; OsCNGC1, Non-selective cation channels 1). OsACTII was used as an internal control ( $n=6$ with 3 replicates). Value represent means $+-\mathrm{SD}$, ns $=$ non-significant, ${ }^{*} p<0.05,{ }^{* *} p<0.01$, and ${ }^{* * *} p<0.001$, two-way ANOVA with Sidak's multiple comparison test.

Figure 7. Whole-genome sequencing (WGS) and RNA-sequencing (RNA-seq) analyses of the sitl1 mutant. (a) Number of sequence variants in the sitl1 mutant compared with wild-type (WT) plant. The SNPs and Indels between the sitl1 mutant and WT were determined via WGS according to their chromosome locations. (b) Characterization of SNP and Indel variants in the sitl1 mutant. Colors in the pie chart represent the different features of variant annotation based on genomic loci. Numbers indicate the number of SNPs and Indels in the sitl1 mutant. (c-d) Transcript abundance ( $\log _{2}$ fold-change) and - $\log _{10}$ qvalue analyses of the genes containing SNP (c) and Indel (d) variants in thesitl1 mutant via RNA-seq. Green and pale green round symbols represent the relative gene expression level and statistical significance, respectively. Gray dot lines indicate the cut-off value of q value $\left(0.05,-\log _{10}\right.$ value of 1.3$)$. Red arrows indicate that genes containing SNPs (c) and Indels (d) in the sitl1 mutant have significantly higher or lower mRNA abundance.

Figure 8. Mutant identification, subcellular localization, and gene expression analyses of OsMTP1 gene. (a) Schematic of the genomic region corresponding to OsMTP1. The position of the $\mathrm{T}$ insertion (red arrows), the initiating codon (ATG), and the stop codon (TAA) are indicated. Genomic OsMTP1 sequences are represented by exons (black), introns (white), and untranslated 5' and 3' UTRs (gray). Green arrow indicates the stop codon in coding sequence (CDS) ofOsMTP1. Blue arrows indicate the qPCR-amplified regions for the gene expression study. (b-c) Relative gene expression analysis of OsMTP1 in roots and leaves of the sitl1 mutant and WT. One-week-old seedlings of the sitl1 mutant and WT were used to determine mRNA abundance of OsMTP1 gene using two different primer pairs of qRT1 (b) and qRT2 (c) via qRT-PCR analysis ( $n=6$ with 3 replicates). OsACTII was used as an internal control. Value represent means $+-\mathrm{SD}$, ns $=$ non-significant, ${ }^{* * *} p<0.001$, two-way ANOva with Sidak's multiple comparison test. (d) Subcellular localization analysis of 35S::OsMTP1-sGFPfusion protein with plasma membrane marker. Left column is the rice protoplast expressing $35 S:: s G F P$ (empty-vector) construct used as a control. Right column is the rice protoplast co-expressing $35 S:: O s M T P 1-s G F P$ fusion protein with pm-rk (plasma membrane marker). (e) Relative gene expression of OsMTP1 in different tissues and development stages in WT. The mRNA abundance of OsMTP1 gene was determined using the qRT2 primer set via qRT-PCR analysis. OsACTII was used as an internal control ( $n=6$ with 3 replicates). Value represent means +- SD. (f-g) Relative gene expression of OsMTP1 in WT under salinity stress conditions. One-week-old seedlings of WT were treated with half-strength KimuraB solution containing 0 or $50 \mathrm{mM} \mathrm{NaCl}$ for $24 \mathrm{~h}$. Root (f) and leaf blade (g) tissues were harvested at $30 \mathrm{~m}, 1 \mathrm{~h}, 6$, and $24 \mathrm{~h}$ after treatments. The mRNA abundance of OsMTP1 gene was determined using the qRT2 primer set via qRT-PCR analysis. OsACTII was used as an internal control ( $n=6$ with 3 replicates). Value represent means + - SD. ns $=$ non-significant, ${ }^{* * *} p<0.001$, two-way AnovA with Sidak's multiple comparison test.

Figure 9. Heterologous overexpression of OsMTP1 increases ability of $\mathrm{Mg}^{2+}$ and $\mathrm{Na}^{+}$transport in yeast. (a-b) The wild-type yeast strain CM52 and CM62 transformed with empty vector (EV) and OE-mOsMTP1 were used as positive and negative controls. (a) Representative images of yeast cell growth on solid medium containing $0,0.1$, and $1 \mathrm{mM} \mathrm{MgCl} 2$. (b) Complementation of yeast cell growth assay in liquid medium containing $0,0.1$, and $1 \mathrm{mM} \mathrm{MgCl}{ }_{2}$. Cell density $\left(\mathrm{OD}_{600}\right)$ of each yeast line was monitored every $2 \mathrm{~h}$ over 66 $\mathrm{h}(n=8$ replicates). Value represent means +- SD. (c-h) Yeast wild-type cells (FM391) harboring EV, OEmOsMTP1, and OE-OsMTP1 was used to monitor cell growth rates in solid and liquid medium containing $0,0.5$, or $1 \mathrm{M}$ of $\mathrm{NaCl}$ and $1 \mathrm{M}$ of $\mathrm{NaCl}$ with 0.1 or $1 \mathrm{mM} \mathrm{MgCl}_{2}$. (c) Representative images of yeast cell growth of WT, EV, OE-mOsMTP1 , and OE-OsMTP1 on solid medium. (d-h) Cell growth assay in liquid medium containing $0 \mathrm{mM}$ (d), $500 \mathrm{mM}$ (e), and $1 \mathrm{M}$ (f) $\mathrm{NaCl}, 1 \mathrm{M} \mathrm{NaCl}$ with $0.1 \mathrm{mM} \mathrm{MgCl}{ }^{2}$ (g) and $1 \mathrm{mM}$ $\mathrm{MgCl}_{2}(\mathrm{~h})$. Cell density $\left(\mathrm{OD}_{600}\right)$ of each yeast line was monitored every $2 \mathrm{~h}$ over $48 \mathrm{~h}$ ( $n=8$ replicates). Value represent means +- SD.

\section{Supplementary figure legends}


Figure S1. Analysis of whole genome sequencing (WGS) and RNA-sequencing quality. (a) WGS data filtered by NGS QC Toolkit. (b) The short reads mapped on the Nipponbare reference genome. (c) RNA-seq data filtered by NGS QC Toolkit.

Figure S2. Assessment of salinity tolerance 100 core collection lines. Seeds of 100 rice mutant lines $\left(\mathrm{M}_{10}\right)$ with wild-type (WT) were germinated and grown in hydroponic solution for 7 days under a 16-h photoperiod. One-week-old seedlings were treated with hydroponic solution containing $100 \mathrm{mM} \mathrm{NaCl}$ for 1 week. Lengths of shoots and roots were measured to evaluate salinity sensitivity with 3 biological replicates. Red lines indicate average lengths of shoot and root of WT plants. Values represent means +- SD, bars with or without asterisks indicate significant difference or non-significant, respectively. ${ }^{*} p<0.05,{ }^{* *} p<0.01$, and ${ }^{* * *} p<0.001$, one-way ANOVA with Sidak's multiple comparison test.

Figure S3. Seed germination rates of wild-type (WT) andsitl1 mutant. Seeds were germinated in hydroponic solution for 7 days under a 16 -h photoperiod. Seed germination rates of WT andsitl1 mutant were scored every day for 7 days ( $n=3$ biological replicates with 50 seeds per replicate)

Figure S4. Alleviation of reduced root growth and leaf chlorophyll content by $\mathrm{Mg}^{2+}$ supply in sitl1 mutant. Rice seeds of sitl1 mutant and wild-type (WT) were germinated and grown in half-strength KimuraB nutrient solution containing $0,10,100$, and $500 \mu \mathrm{M} \mathrm{Mg}^{2+}$ for 7 days. (a) Representative seedling images of sitl1 mutant and WT plants exposed to nutrient solution containing 0 or $500 \mu \mathrm{M} \mathrm{Mg}^{2+}$. (b) Comparison of fresh weight of root ( $n=30$ with 3 replicates). (c) Fresh weight of shoot $(n=30$ with 3 replicates). (d) Representative leaf images of sitl1 mutant and WT exposed to a nutrient solution containing $500 \mu \mathrm{M} \mathrm{Mg}^{2+}$. (e) Comparison of total chlorophyll content of leaves of sitl1 mutant and WT ( $n=6$ with 3 replicates). Value represent means $\pm \mathrm{SD}$, ns $=$ non-significant, ${ }^{*} p<0.05,{ }^{* *} p<0.01$, and ${ }^{* * *} p<0.001$, two-way ANOVA with Sidak's multiple comparison test.

Figure S5. Assessment of salinity and drought tolerance of sitl1 mutant. Rice seeds of sitl1 mutant and wild-type (WT) were germinated and grown in soil mix for 7 days under a 16-h photoperiod. For the salinity treatment, one-week-old seedlings were irrigated with half-strength nutrient solution containing 0 or $50 \mathrm{mM} \mathrm{NaCl}$ for 2 weeks. For the drought treatment, one-week-old seedlings were withheld for 7 days and re-watered for 7 days. (a) Representative images of sitl1 mutant and WT plants exposed to nutrient solution containing 0 (control), $50 \mathrm{mM} \mathrm{NaCl}$ (salinity) or drought stress. (b) Comparison of fresh weight of shoot under normal growth condition ( $n=30$ with 3 replicates). (c) Fresh weight of shoot under salinity stress condition ( $n=30$ with 3 replicates). (d) Fresh weight of shoot under drought stress condition $(n=$ 30 with 3 replicates). Value represent means $+-\mathrm{SD}, \mathrm{ns}=$ non-significant, ${ }^{* * *} p<0.001$, Student's t-test.

Figure S6. Heat map analysis and relative fold expression of the genes encoding antioxidant defense enzymes, $\mathrm{Na}^{+}$, and $\mathrm{K}^{+}$transporters in sitl1 mutant. One-week-old seedlings of sitl1 mutant and wild-type (WT) were used to sample leaf and root tissues. (a) Heat map of genes encoding antioxidant defense enzymes, $\mathrm{Na}^{+}$and $\mathrm{K}^{+}$transporters in roots of sitl1 and WT ( $n=3$ replicates). Values of $\log _{2}$ fold-change and q-value were obtained from the RNA-sequencing analyses. (b) Relative expression levels of selected genes encoding antioxidant defense enzymes in roots and leaves of sitl1 and WT $(n=6$ with 3 replicates). (c) Relative expression levels of selected genes encoding $\mathrm{Na}^{+}$and $\mathrm{K}^{+}$transporters in roots and leaves of sitl1 and WT ( $n=6$ with 3 replicates). Value represent means $+-\mathrm{SD}$, ns $=$ non-significant, ${ }^{*} p<$ $0.05,{ }^{* *} p<0.01$, and ${ }^{* * *} p<0.001$, two-way ANOVA with Sidak's multiple comparison test.

Figure S7. Shared variants of sitl1 mutant and WT via whole-genome sequencing (WGS) analysis. One-week-old seedlings of sitl1 mutant and wild-type (WT) were used to determine shared variants of sitl1 mutant and WT. The shared SNPs and Indels between sitl1 mutant and WT (Donganbyeo) were determined based on rice reference genome (Nipponbare) according to their genome locations.

Figure S8. Scatter dot plot of DEGs in the sitl1 mutant.Illumina-based RNA-seq was performed to profile mRNA expression in roots of one-week-old seedlings of the sitl1 mutant and wild-type (WT). DEGs with statistical significance were obtained $(n=3$ replicates, q value $<0.05)$. A set of 767 and 438 genes was identified whose mRNAs showed significantly increased and decreased transcript abundance, respectively, in 
roots in the sitl1 mutant.

Figure S9. The over-represented gene functions of differentially expressed genes (DEGs) in the sitl1 mutant. Pageman analysis of the sitl1 mutant versus WT in roots. The different colors represent the degree of change in the gene expression level (log2 fold change) according to Fisher's exact test with default parameter. Red represents the significant enrichment of DEGs, blue represents the significant depletion of DEGs, and white represents no significance.

Figure S10. Metabolism overview of differentially expressed genes (DEGs) in the sitl1 mutant. Blue or Red colors represent mRNA expression levels ( $\log _{2}$ fold change) of the upregulated or downregulated genes, respectively, in the sitl1 mutant.

Figure S11. A protein sequence alignment of OsMTP1 in WT and thesitl1 mutant. OsMTP1 sequences were confirmed by rice cDNA sequences in WT and the sitl1 mutant. Alignment was performed using CLC Main Workbench software ver. 8.0.1 (https://digitalinsights.qiagen.com/products-overview/analysisand-visualization/qiagen-clc-main-workbench/). The putative metal ion transporter CorA-like cation transporter domain was marked by red rectangles. Predicted two C-terminal transmembrane (TM) domains were indicated as green arrows. Red arrow indicates T insertion (1044_1045insT) and blue arrow indicates predicted STOP codon of OsMTP1 in sitl1 mutant. The domain structure of the OSMTP1 protein was predicted using the InterproScan server (https://www.ebi.ac.uk/interpro/). The present of TM domain was predicted using InterProScan and TMHMM server v.2.0 (http://www.cbs.dtu.dk/services/TMHMM/).

Figure S12. Phylogenetic tree of O. sativa OsMTP1 and its orthologous genes . (a) Protein sequences of OsMTP1 and its orthologous genes in A. thaliana, Z. mays, and S. bicolor . Multiple alignment was performed using CLC Main Workbench software ver. 8.0.1 (https://digitalinsights.qiagen.com/productsoverview/analysis-and-visualization/qiagen-clc-main-workbench/). The putative metal ion transporter CorAlike cation transporter domain was marked by red rectangles. Predicted two C-terminal transmembrane (TM) domains were indicated as green rectangles. (b) A Neighbor-Joining tree of protein sequences constructed with CLC Main Workbench software ver. 8.0.1 (https://digitalinsights.qiagen.com/products-overview/analysisand-visualization/qiagen-clc-main-workbench/). Asterisk indicates OsMTP1. Bootstrap analysis was performed with 1,000 replicates. Bootstrap percentages are indicated at branches. The domain structure of the OSMTP1 protein was predicted using the InterproScan server (https://www.ebi.ac.uk/interpro/). The present of TM domain was predicted using InterProScan and TMHMM server v.2.0 (http://www.cbs.dtu.dk/services/TMHMM

Figure S13. A protein sequence alignment of $O$. sativa and A. thaliana MRS2 proteins with OsMTP1 (and its orthologous genes) in O. sativa, A. thaliana, Z. mays, and S. bicolor. Protein sequences of MRS2 family and OsMTP1 orthologous genes in O. sativa, A. thaliana, and Z. mays were obtained from The Arabidopsis Information Resource (TAIR,https://www.arabidopsis.org/) and phytozome 12 (https://phytozome.jgi.doe.gov/pz/portal.html). A Neighbor-Joining tree of protein sequences constructed with CLC Main Workbench software ver. 8.0.1 (https://digitalinsights.qiagen.com/productsoverview/analysis-and-visualization/qiagen-clc-main-workbench/). Red box indicates GM(I)N-motif in the first transmembrane domain. Bootstrap analysis was performed with 1,000 replicates. Bootstrap percentages are indicated at branches.

Figure S14. Phylogenetic tree of $O$. sativa and A. thaliana MRS2 proteins with OsMTP1 (and its orthologous genes) inO. sativa, A. thaliana, Z. mays, and S. bicolor. Protein sequences of MRS2 family and OsMTP1 orthologous genes in O. sativa, A. thaliana, and Z. mays were obtained from The Arabidopsis Information Resource (TAIR,https://www.arabidopsis.org/) and phytozome 12 (https://phytozome.jgi.doe.gov/pz/portal.html). A Neighbor-Joining tree of protein sequences constructed with CLC Main Workbench software ver. 8.0.1 (https://digitalinsights.qiagen.com/products-overview/analysisand-visualization/qiagen-clc-main-workbench/). Asterisk indicates OsMTP1. Bootstrap analysis was performed with 1,000 replicates. Bootstrap percentages are indicated at branches.

Supplementary Table information 
Supplementary Table S1. List of primers used in this study.

Supplementary Table S2. A total of 438 upregulated DEGs with stastical significance in the sitl1 mutant (q value $<0.05, n=3$ replicates).

Supplementary Table S3. A total of 767 downregulated DEGs with stastical significance in the sitl1 mutant (q value $<0.05, n=3$ replicates).
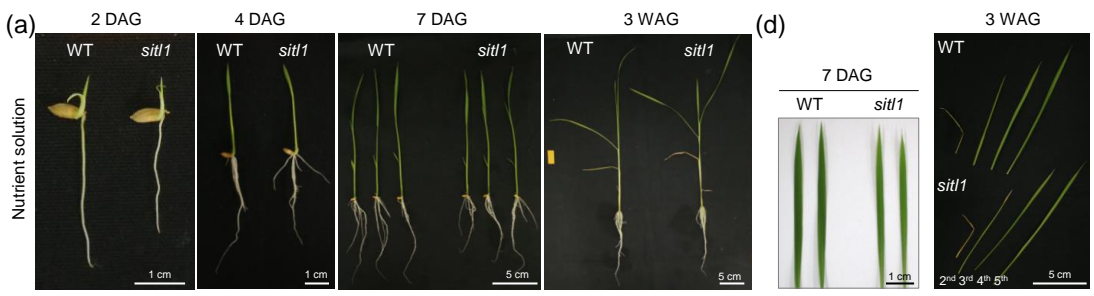

(b)
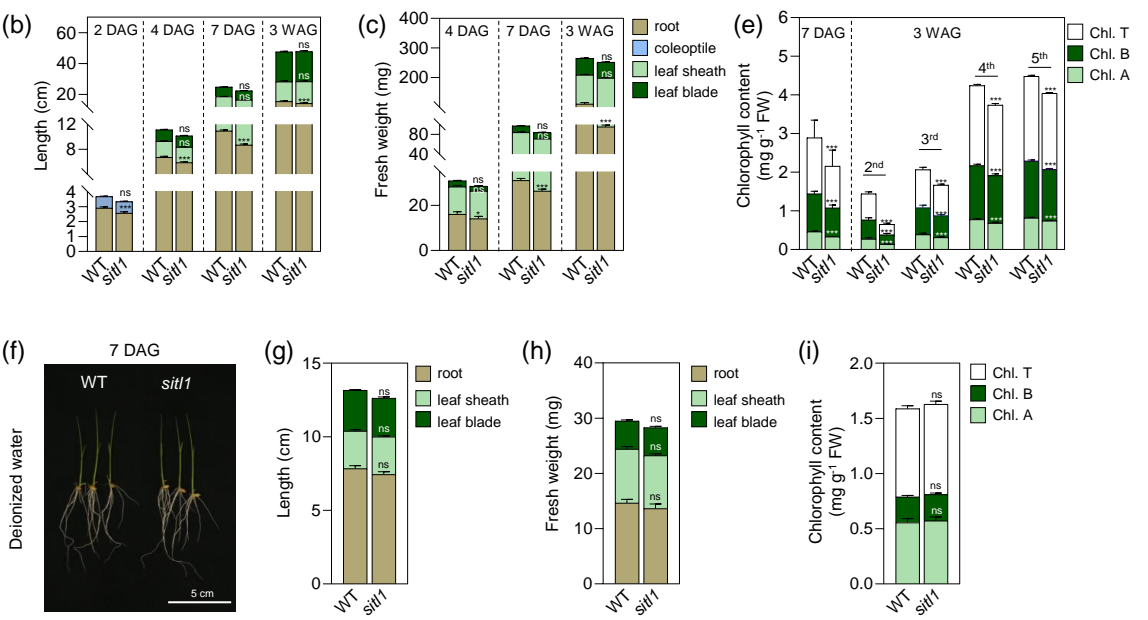

Figure 1. sit/1 mutant decreases root growth and leaf chlorophyll content. Rice seeds of the sit/1 mutant and wildtype (WT) control were germinated and grown in half-strength KimuraB nutrient solution or deionized water for 3 weeks (a) Representative seedling images of the sitl1 mutant and WT plants at 2, 4, and 7 days after germination (DAG) and 3 weeks after germination (WAG) under nutrient solution condition. (b) Comparison of lengths of root, coleoptile, leaf sheath, and leaf blade ( $n=30$ with 3 replicates). (c) Fresh weight ( $n=30$ with 3 replicates). (d) Representative leaf images of the sitl1 mutant and WT plants at 7 DAG and 3 WAG. (e) Comparison of leaf chlorophyll content ( $n=6$ replicates). Leaves were sampled and measured total chlorophyll, chlorophyll $\mathrm{A}$, and chlorophyll B at 7 DAG and 3 WAG. (f) Representative seedling images of the sit/1 mutant and WT plant at 7 DAG under deionized water condition. Seeds of the sit/1 mutant and WT were germinated and grown in deionized water for 7 days. (g) Comparison of lengths of root, leaf sheath, and leaf blade at 7 DAG ( $n=30$ with 3 replicates). (h) Fresh weight ( $n=30$ with 3 replicates). (i) Leaf chlorophyll content ( $n=6$ replicates). Value represent means \pm SD, ns $=$ non-significant, ${ }^{*} p<0.05$ and ${ }^{* *} p<0.001$, two-way ANOVA with Sidak's multiple comparison test. 

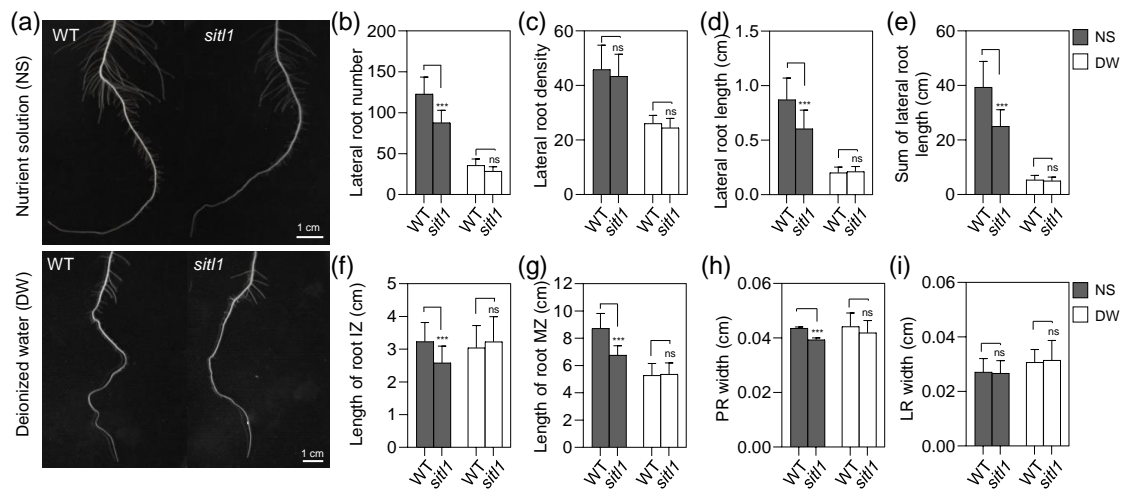

Figure 2. Analysis of the sit/1 mutant in root development. Rice seeds of the sit/1 mutant and wild-type (WT) control were germinated and grown in half-strength KimuraB nutrient solution (NS) or deionized water (DW) for 1 week.

Detached roots were used to scan and measure for root development. (a) Representative images of 1 -week-old stil1 mutant and WT roots. (b) Comparison of lateral root number in primary roots ( $n=30$ with 3 replicates). (c) Lateral root density ( $n=30$ with 3 replicates). (d) Average length of lateral root $(n=30$ with 3 replicates). (e) Sum of lateral root length ( $n=30$ with 3 replicates). Lateral root number, average length of lateral root, and sum of lateral root length were analyzed within $3-\mathrm{cm}$ root samples from root base. (f) Length of root immature zone ( $n=30$ with 3 replicates). Root immature zone was defined as the zone of the root where lateral roots were not detected from the root tip. $(\mathrm{g})$ Length of root mature zone ( $n=30$ with 3 replicates). Root mature zone was defined as the zone of the root where lateral root initiation and development takes places to root base. (h) Length of primary root (PR) width ( $n=30$ with 3 replicates). (i) Length of lateral root width ( $n=30$ with 3 replicates). Value represent means $\pm S D, n s=n$ non-significant, ${ }^{* * *} p<0.001$, two-way ${ }_{\text {ANOVA }}$ with Sidak's multiple comparison test. 

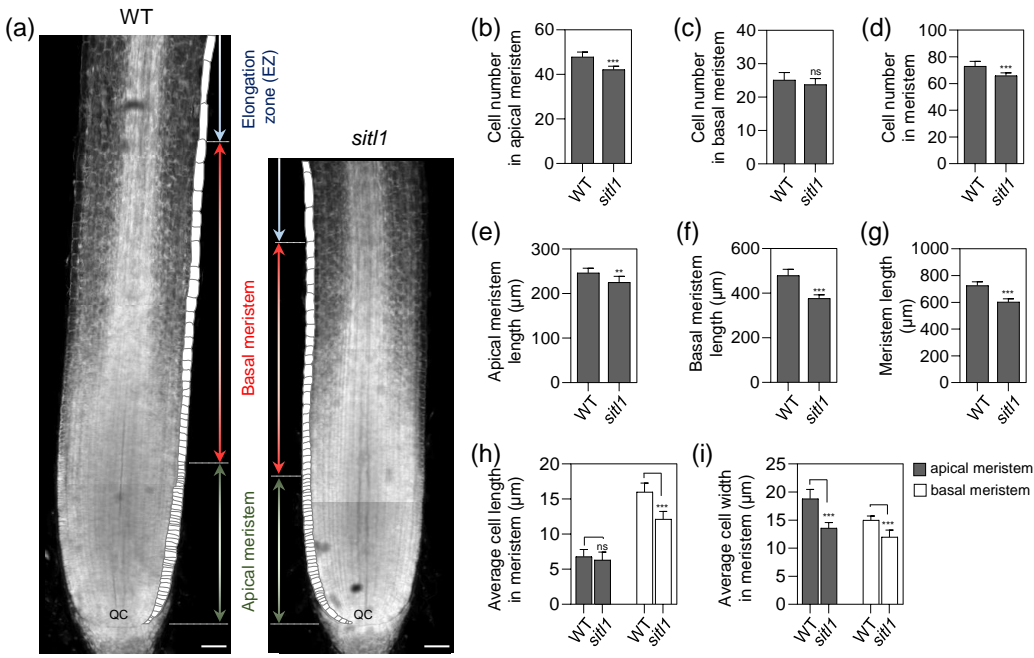

(e)

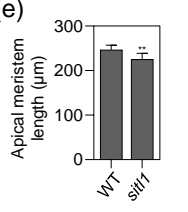

(f)

(g)
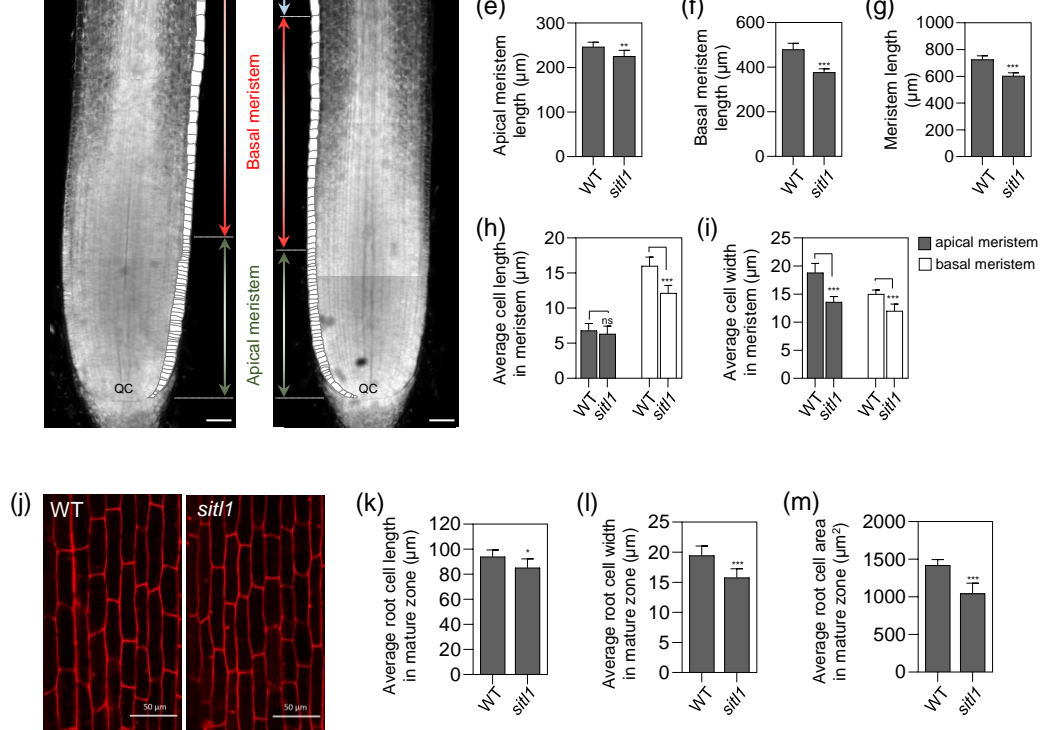

(k)

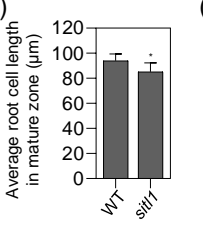

(I)
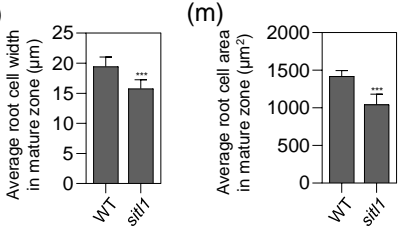

Figure 3. sitl1 mutant decreases cell number and size in root tissue. Rice seeds of the sitl1 mutant and wild-type (WT) were germinated and grown in half-strength KimuraB solution for 1 week. One-week-old roots were detached and stained with propidium iodide (PI) solution for $10 \mathrm{~min}$. Root images were captured via confocal laser-scanning microscopy and the epidermal cells were used to measure cell size and number. (a) Representative images of root meristems of the sit/1 mutant and WT. The root epidermal cells are outlined with solid lines. Green, red, and blue lines with double arrowheads represent the lengths of the apical meristem, the basal meristem and the

elongation/differentiation zone, respectively (Hacham et al., 2011; Lim et al., 2018). QC indicates the quiescent center. Scale bar, $40 \mu \mathrm{m}$. Quantification of (b) average cell number in the apical meristem, (c) cell number in the basal meristem and (d) total cell number in the meristem zone ( $n=9$ with 3 replicates). Quantification of (e) apical meristem length, $(\mathrm{f})$ basal meristem length and $(\mathrm{g})$ total meristem length $(n=9$ with 3 replicates). Quantification of $(\mathrm{h})$ average cell length and (i) cell width in the apical and basal meristems ( $n=9$ with 3 replicates). (j) Representative images of cortical cells in mature root zone of the sit/1 mutant and WT. Quantification of average (k) cortical cell length, (l) cell width and $(\mathrm{m})$ cell area in the mature root zone $(n=9$ with 3 replicates). Value represent means $\pm \mathrm{SD}, \mathrm{ns}=$

non-significant, ${ }^{*} p<0.05,{ }^{* *} p<0.05$ and ${ }^{* *} p<0.001$, Student's $t$-test (b, c, d, e, f, g, k, l, and $\mathrm{m}$ ) and two-way ANOva with Sidak's multiple comparison test (h and i). 


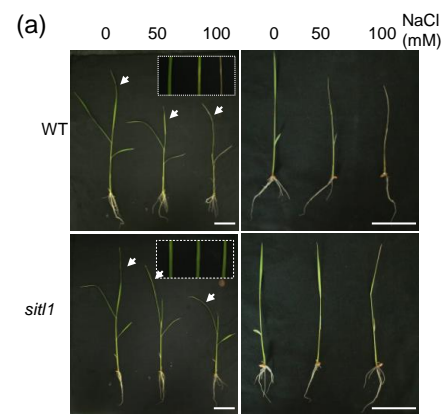

Nutrition solution (NS) Deionized water (DW)

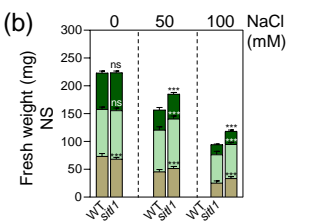

(d)

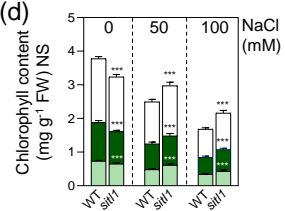

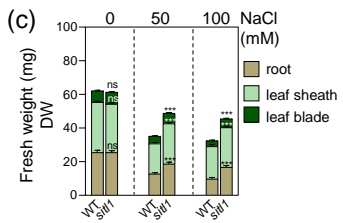

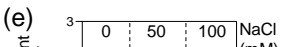

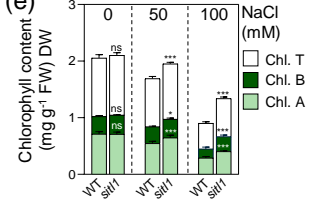

(f)

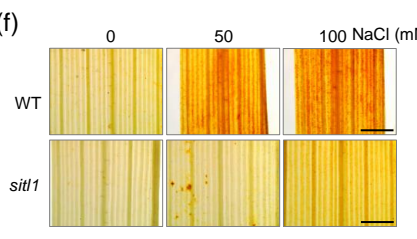

Nutrient solution (NS)
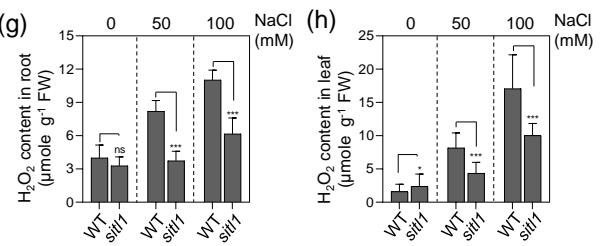

(i)
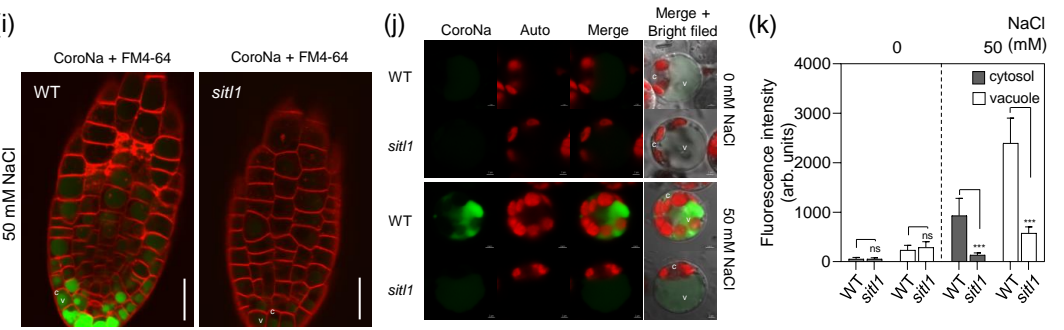

Figure 4. sit/1 mutant enhances salinity insensitivity by reducing $\mathrm{Na}+$ influx across the plasma membrane. Rice seeds of the sit/1 mutant and wild-type (WT) were grown for 1 week under half-strength KimuraB nutrient solution (NS) or deionized water (DW) conditions. Seedlings were then treated with NS or DW containing $0,50,100 \mathrm{mM} \mathrm{NaCl}$ for 1 week. (a) Representative images of the sitl1 mutant and WT at 7 days after salinity treatment. Boxes with broken lines indicate the third leaf of the sit/1 mutant and WT. Scale bar, $5 \mathrm{~cm}$. Quantification of fresh weights of root, leaf sheath, and leaf blade tissues under (b) NS condition and (c) DW condition ( $n=30$ with 3 replicates). Quantification of leaf chlorophyll content under (d) NS condition and (e) condition ( $n=30$ with 3 replicates). ( $f$ ) Representative DAB staining images of leaf blades of the sit/1 mutant and WT. Rice seeds of the sit/1 mutant WT were germinated and grown in halfstrength NS for 1 week. Seedlings were then treated with NS containing $0,50,100 \mathrm{mM} \mathrm{NaCl}$ for 1 week and leaf blades were stained with DAB solution to assess the accumulation of $\mathrm{H}_{2} \mathrm{O}_{2}$. Scale bar, $1 \mathrm{~mm}$. (g) Quantification of $\mathrm{H}_{2} \mathrm{O}_{2}$ content in root tissues ( $n=6$ with 3 replicates). (h) Quantification of $\mathrm{H}_{2} \mathrm{O}_{2}$ content in leaf blade tissues ( $n=6$ with 3 replicates). (i) Representative images of $\mathrm{Na}^{+}$accumulations in lateral roots of the sit/1 mutant and WT. One-week-old seedlings of the sit/1 mutant and WT were treated with NS containing $50 \mathrm{mM} \mathrm{NaCl}$ for $3 \mathrm{~h}$. The lateral roots were detached and stained with CoroNa-green AM and FM4-64 to visualize the accumulations of $\mathrm{Na}^{+}$in the vacuole. (j) Representative images of $\mathrm{Na}$ + distribution in rice protoplasts of the sit/1 mutant and WT. Leaf protoplasts were isolated and treated with 0 and $50 \mathrm{mM} \mathrm{NaCl}$ solution for $1 \mathrm{~h}$. The CoroNa-green AM was used to visualize $\mathrm{Na}^{+}$distribution in protoplasts. (k) Quantification of CoroNa green intensity ( $n=3$ replicates with average intensity of 50 protoplasts per replicate). Value represent means $\pm \mathrm{SD}, \mathrm{ns}=$ non-significant, ${ }^{*} p<0.05$ and ${ }^{* * *} p<0.001$, two-way ANOvA with Sidak's multiple comparison test. 

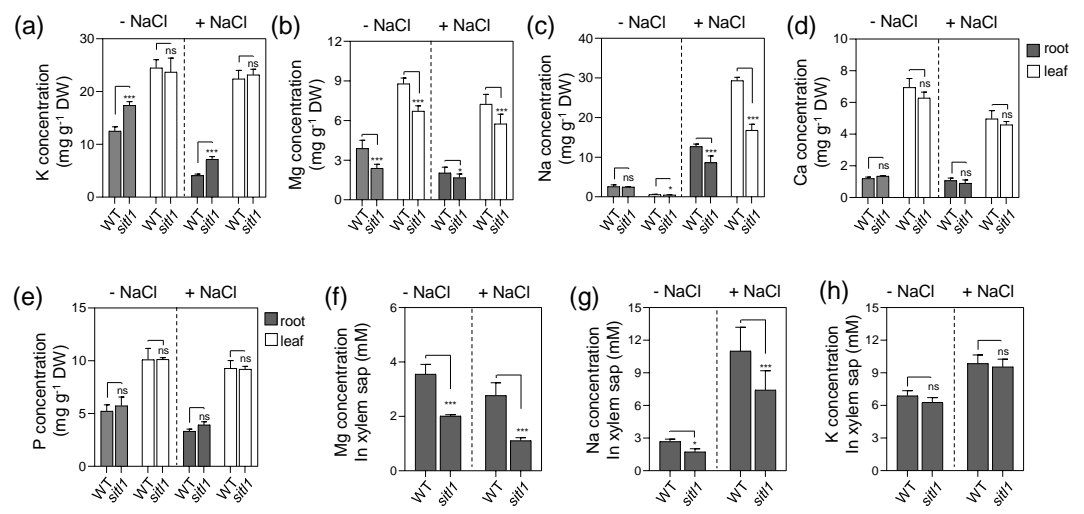

Figure 5. sitl1 mutant reduces the concentration of $\mathrm{Mg}^{2+}$ and $\mathrm{Na}^{+}$in root and leaf tissues. One-week-old rice seedlings of the sit/1 mutant and wild-type (WT) were treated with 0 or $50 \mathrm{mM} \mathrm{NaCl}$ solution for 1 week. Oven-dried root and leaf samples of the sit/1 mutant and WT were used to determine the concentrations of inorganic ions via ICP-OES $(n=3$ replicates). (a) Potassium. (b) Magnesium. (c) Sodium. (d) Calcium. (e) Phosphorus. Xylem sap was collected from 1week-old rice seedlings of the sitl1 mutant and WT after 0 or $50 \mathrm{mM} \mathrm{NaCl}$ treatments. Collected sap was used to

determine the concentrations of inorganic ions via ICP-OES ( $n=6$ replicates). Quantification of (f) Magnesium, $(\mathrm{g})$

Sodium, and (h) Potassium concentrations in xylem sap. Value represent means $\pm \mathrm{SD}$, ns $=$ non-significant, ${ }^{*} p<0.05$ and ${ }^{* * *} p<0.001$, two-way ANOva with Sidak's multiple comparison test. 
(a)
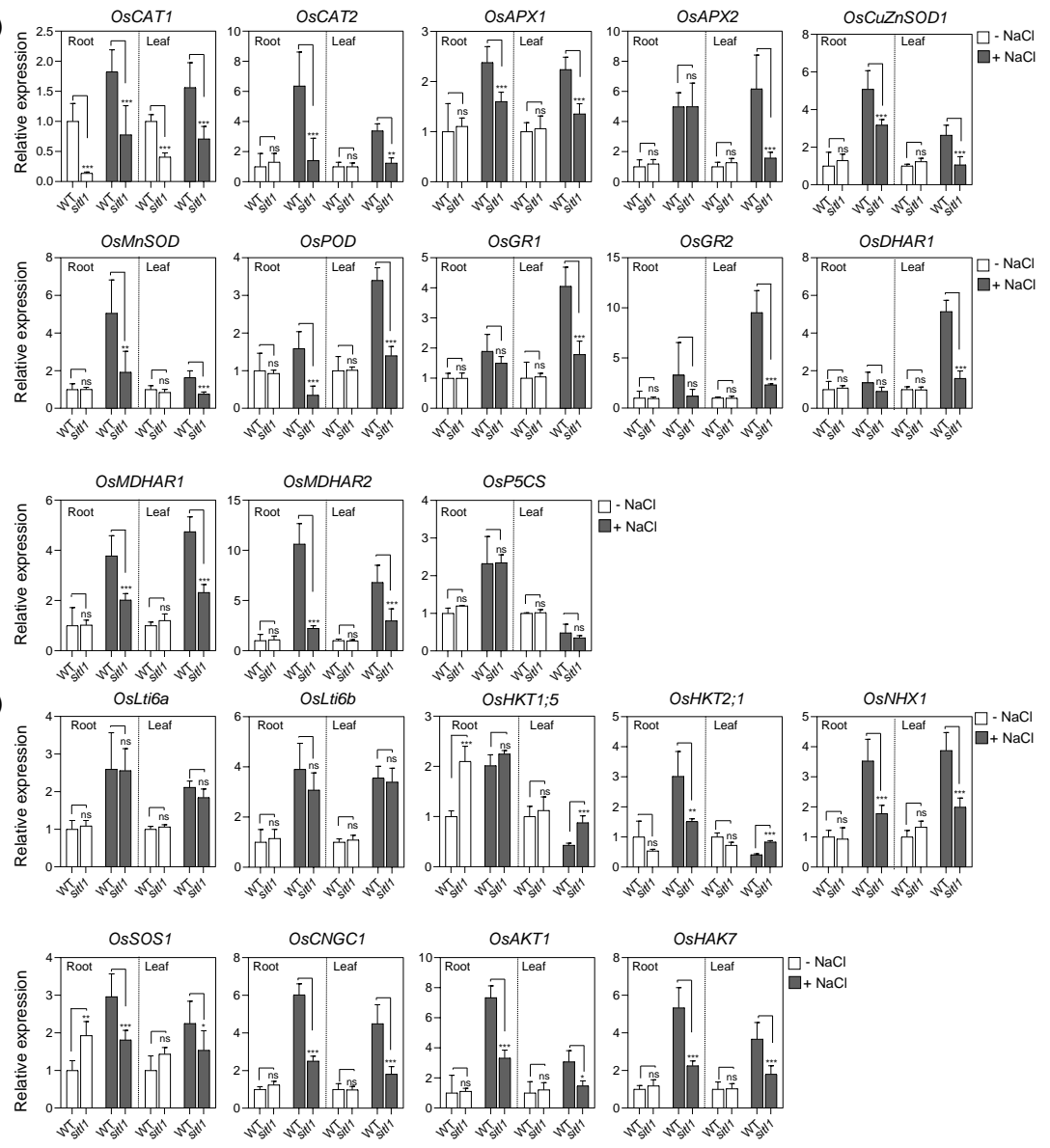

Figure 6. Relative fold expression of the genes encoding antioxidant defense enzymes, $\mathrm{Na}^{+}$, and $\mathrm{K}^{+}$transporters. Oneweek-old seedlings of the sit/1 mutant and WT were treated with half-strength KimuraB solution containing 0 or $50 \mathrm{mM}$ $\mathrm{NaCl}$ for $1 \mathrm{~h}$. Relative expression levels of selected marker genes in root and leaf tissues were determined by qRTPCR. (A) Relative expression of the genes encoding antioxidant defense enzymes (OsCAT1, Catalase isozyme A; OsCAT2, Catalase isozyme B; OsAPX1, Cytosolic ascorbate peroxidase 1; OsAPX2, Cytosolic ascorbate peroxidase 2; OsCuZnSOD1, Cytosolic copper/zinc-superoxide dismutase 1; OsMnSOD, Mitochondrial manganese-superoxide dismutase; OsPOD, Peroxidase; OsGR1, Cytosolic glutathione reductase 1; OsGR2, Mitochondrial glutathione reductase; OsDHAR1, Dehydroascorbate reductase; OsMDHAR1, Cytosolic monodehydroascorbate reductase reductase; OsDHAR1, Dehydroascorbate reductase; OSMDHAR1, Cytosolic monodehydroascorbate reductase;
OsMDHAR2, Putative monodehydroascorbate reductase; OsP5CS, Delta-1-pyrroline-5-carboxylate synthase). (B) Relative expression of the genes encoding $\mathrm{Na}^{+}$and $\mathrm{K}^{+}$transporters (OsHKT1;5, Sodium transporter Hkt1.5; OsLti6a, Plasma membrane protein 3 homolog; OsLti6b, Plasma membrane protein 3 homolog; OsHKT2;1, High-affinity potassium transporter; OsNHX1, Vacuolar $\mathrm{Na}^{+} / \mathrm{H}^{+}$antiporter; OsSOS1, Salt overly sensitive 1; OsAKT1, AKT-type $\mathrm{K}^{+}$ channels; HAK7, Potassium transporter 7; OsCNGC1, Non-selective cation channels 1). OsACTI/ was used as an internal control ( $n=6$ with 3 replicates). Value represent means $\pm \mathrm{SD}, \mathrm{ns}=$ non-significant, ${ }^{*} p<0.05,{ }^{* *} p<0.01$, and ${ }^{* *} p<0.001$, two-way ANOva with Sidak's multiple comparison test. 


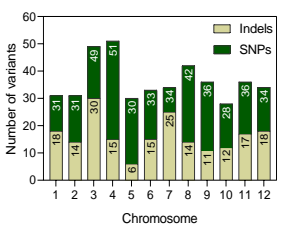

(c) SNPs in sitt1 mutant

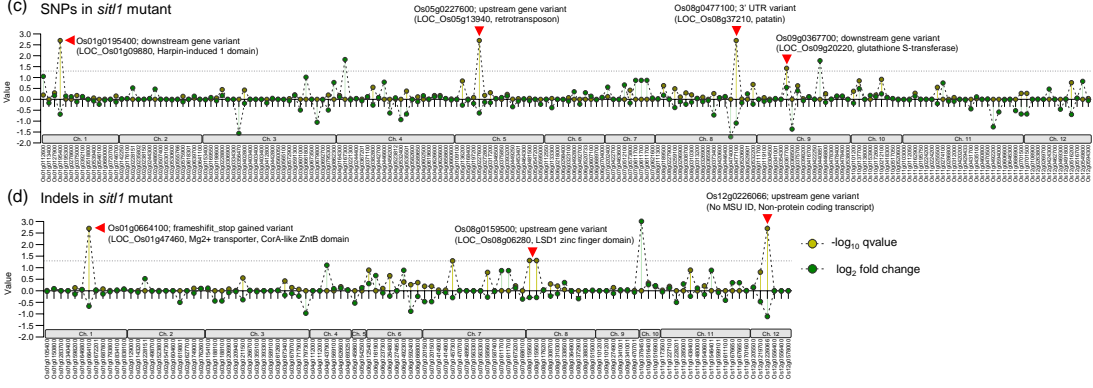

Figure 7. Whole-genome sequencing (WGS) and RNA-sequencing (RNA-seq) analyses of the sitt1 mutant. (a) Number of sequence variants in the sitt1 mutant compared with wild-type (WT) plant. The SNPs and Indels between the sittl mutant and WT were determined via WGS according to their chromosome locations. (b) Characterization of SNP and Indel variants in the sittl mutant. Colors in the pie chart represent the different features of variant annotation based on genomic loci. Numbers indicate the number o
SNPS and Indels in the sitt' mutant. (c-d) Transcript abundance (log fold-change) and -log log $_{10}$ qvalue analyses of the genes containing SNP (c) and Indel (d) variants in the sittl mutant via RNA-s
the cut-off value of $q$ vallat the cut-off value of q value $\left(0.05,-\log _{10}\right.$ value of 1.3$)$. Red arrows indicate that genes containing SNPs (c) and Indels (d) in the sitt 1 mutant have significantly higher or lower mRNA abundance.

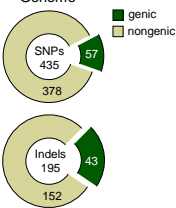

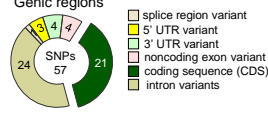

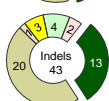

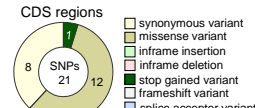

$\underbrace{\operatorname{Indels}}_{1} \frac{1}{13}$

splice acceptor variant

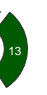

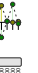

(b) Genome

Genic regions

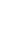


(a) $\mathrm{Mg}^{2+}$ transporter protein, CorA-like ZntB cation

DNA
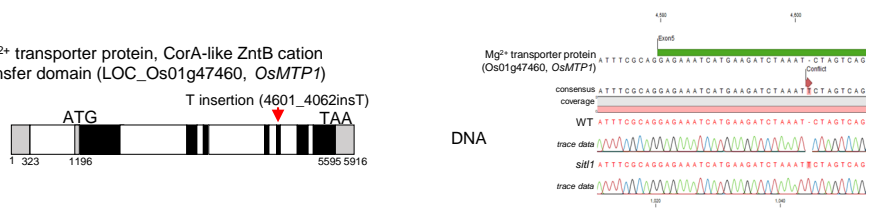

CDS
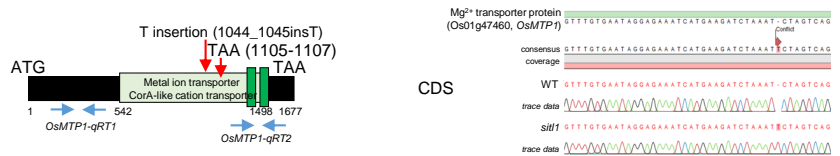

(b)

$\square$ root $\square$ leaf

(c)
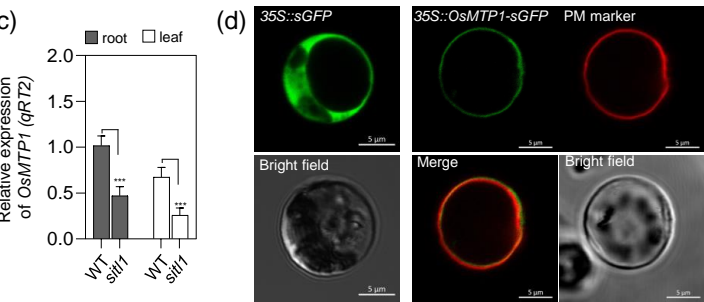

(e)
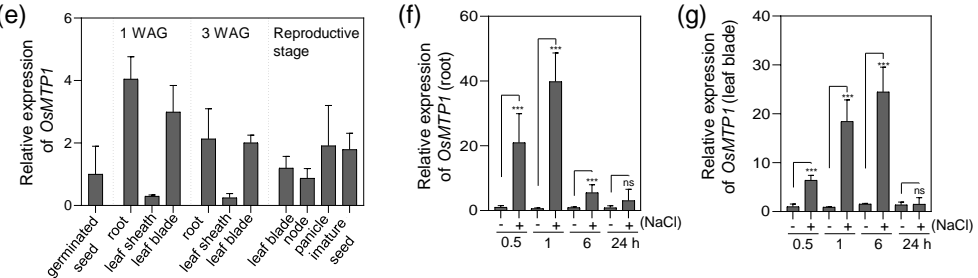

Figure 8. Mutant identification, subcellular localization, and gene expression analyses of OsMTP1 gene. (a) Schematic of the genomic region corresponding to OSMTP1. The position of the T insertion (red arrows), the initiating codon (ATG), and the stop codon (TAA) are indicated. Genomic OsMTP1 sequences are represented by exons (black), introns (white), and untranslated 5' and 3' UTRs (gray). Green arrow indicates the stop codon in coding sequence (CDS) of OsMTP1. Blue arrows indicate the qPCR-amplified regions for the gene expression study. (b-c) Relative gene expression analysis of OSMTP1 in roots and leaves of the sitl1 mutant and WT. One-week-old seedlings of the sit/1 mutant and WT were used to determine mRNA abundance of OsMTP1 gene using two different primer pairs of qRT1 (b) and qRT2 (c) via qRT-PCR analysis ( $n=6$ with 3 replicates). OsACTIl was used as an internal control. Value represent means $\pm \mathrm{SD}$, ns $=$ non-significant, ${ }^{* * \star} p<0.001$, two-way ${ }_{\text {ANOVA }}$ with Sidak's multiple comparison test. (d) Subcellular localization analysis of 35S::OSMTP1-sGFP fusion protein with plasma membrane marker. Left column is the rice protoplast expressing 35S::SGFP (empty-vector) construct used as a control. Right column is the rice protoplast coexpressing 35S::OSMTP1-sGFP fusion protein with pm-rk (plasma membrane marker). (e) Relative gene expression of OSMTP1 in different tissues and development stages in WT. The mRNA abundance of OsMTP1 gene was determined using the qRT2 primer set via qRT-PCR analysis. OsACTI/ was used as an internal control ( $n=6$ with 3 replicates). Value represent means \pm SD. (f-g) Relative gene expression of OsMTP1 in WT under salinity stress conditions. Oneweek-old seedlings of WT were treated with half-strength KimuraB solution containing 0 or $50 \mathrm{mM} \mathrm{NaCl}$ for $24 \mathrm{~h}$. Root (f) and leaf blade $(\mathrm{g})$ tissues were harvested at $30 \mathrm{~m}, 1 \mathrm{~h}, 6$, and $24 \mathrm{~h}$ after treatments. The mRNA abundance of OsMTP1 gene was determined using the qRT2 primer set via qRT-PCR analysis. OsACTI/ was used as an internal control ( $n=6$ with 3 replicates). Value represent means \pm SD. $\mathrm{ns}=$ non-significant, ${ }^{* *} p<0.001$, two-way ANOVA with Sidak's multiple comparison test. 
(a)

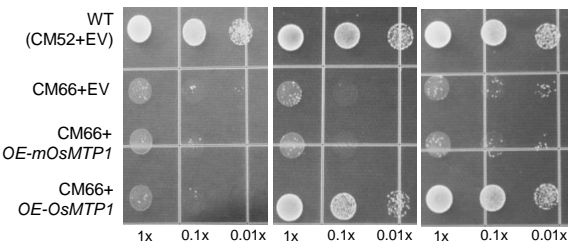

(b) $\rightarrow-\mathrm{WT}(\mathrm{CM} 52+\mathrm{EV}) \rightarrow \mathrm{CM} 66+\mathrm{EV}) \rightarrow \mathrm{OE}-\mathrm{MOSMTP1}_{\mathrm{OE}}^{\mathrm{CM}-\mathrm{OSMTP}}$

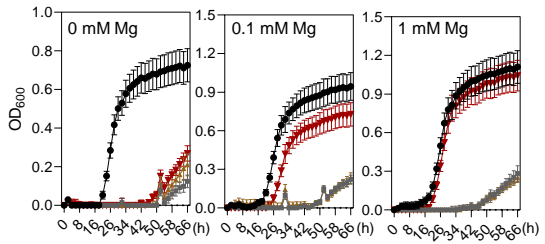

(c)
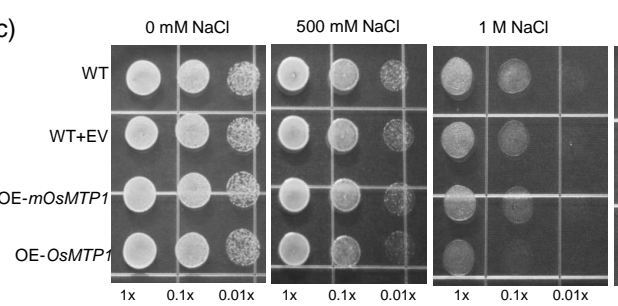

$\begin{aligned} & 1 \mathrm{M} \mathrm{NaCl} \\ + & 0.1 \mathrm{mM} \mathrm{Mg}\end{aligned}$

$1 \mathrm{M} \mathrm{NaCl}$
$+1 \mathrm{mM} \mathrm{Mg}$
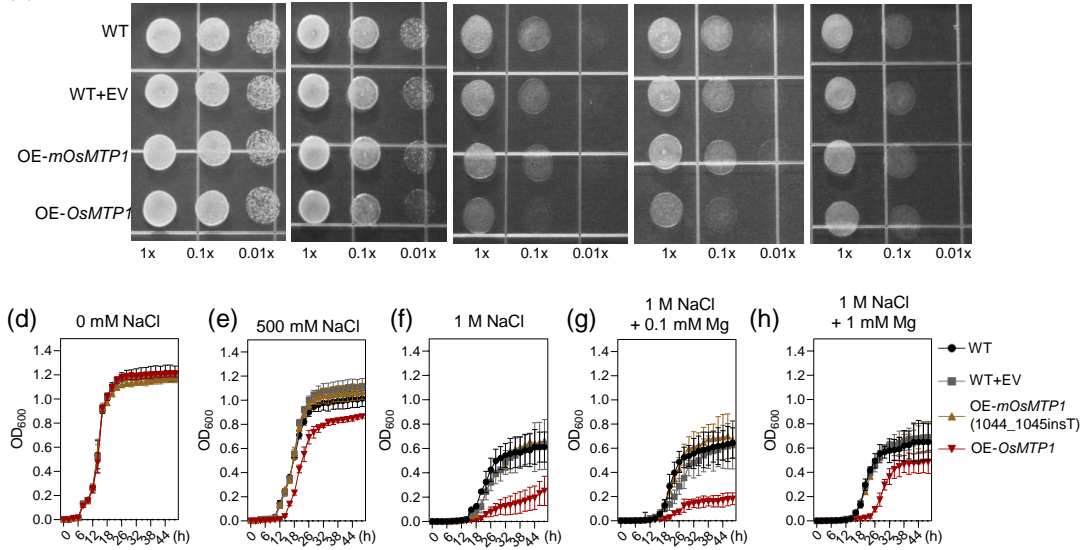

Figure 9. Heterologous overexpression of OsMTP1 increases ability of $\mathrm{Mg}^{2+}$ and $\mathrm{Na}^{+}$transport in yeast. (a-b) The wildtype yeast strain CM52 and CM62 transformed with empty vector (EV) and OE-mOsMTP1 were used as positive and negative controls. (a) Representative images of yeast cell growth on solid medium containing $0,0.1$, and $1 \mathrm{mM} \mathrm{MgCl}_{2}$. (b) Complementation of yeast cell growth assay in liquid medium containing $0,0.1$, and $1 \mathrm{mM} \mathrm{MgCl}_{2}$. Cell density $\left(\mathrm{OD}_{600}\right)$ of each yeast line was monitored every $2 \mathrm{~h}$ over $66 \mathrm{~h}(n=8$ replicates). Value represent means $\pm \mathrm{SD}$. (c-h) Yeast wild-type cells (FM391) harboring EV, OE-mOsMTP1, and OE-OsMTP1 was used to monitor cell growth rates in solid and liquid medium containing $0,0.5$, or $1 \mathrm{M}$ of NaCl and $1 \mathrm{M}$ of $\mathrm{NaCl}$ with 0.1 or $1 \mathrm{mM} \mathrm{MgCl}_{2}$. (c) Representative images of yeast cell growth of WT, EV, OE-mOSMTP1, and OE-OSMTP1 on solid medium. (d-h) Cell growth assay in liquid medium containing $0 \mathrm{mM}$ (d), $500 \mathrm{mM}(\mathrm{e})$, and $1 \mathrm{M}$ (f) $\mathrm{NaCl}, 1 \mathrm{M} \mathrm{NaCl}$ with $0.1 \mathrm{mM} \mathrm{MgCl}^{2}$ (g) and $1 \mathrm{mM} \mathrm{MgCl}_{2}(\mathrm{~h})$. Cell density $\left(\mathrm{OD}_{600}\right)$ of each yeast line was monitored every $2 \mathrm{~h}$ over $48 \mathrm{~h}(n=8$ replicates). Value represent means \pm SD. 